\title{
The Origin of Chern-Simons Modified Gravity from an 11 + 3-Dimensional Manifold
}

\author{
J. A. Helayël-Neto, ${ }^{1}$ Alireza Sepehri, ${ }^{2}$ and Tooraj Ghaffary ${ }^{3}$ \\ ${ }^{1}$ Centro Brasileiro de Pesquisas Fisicas, Rua Dr. Xavier Sigaud 150, Urca, RJ 22290-180, Brazil \\ ${ }^{2}$ Research Institute for Astronomy and Astrophysics of Maragha (RIAAM), P.O. Box 55134-441, Maragha, Iran \\ ${ }^{3}$ Department of Science, Shiraz Branch, Islamic Azad University, Shiraz, Iran
}

Correspondence should be addressed to J. A. Helayël-Neto; helayel@cbpf.br

Received 14 April 2017; Accepted 25 September 2017; Published 20 December 2017

Academic Editor: Piero Nicolini

Copyright ( 92017 J. A. Helayël-Neto et al. This is an open access article distributed under the Creative Commons Attribution License, which permits unrestricted use, distribution, and reproduction in any medium, provided the original work is properly cited. The publication of this article was funded by SCOAP S $^{3}$

\begin{abstract}
It is our aim to show that the Chern-Simons terms of modified gravity can be understood as generated by the addition of a 3dimensional algebraic manifold to an initial 11-dimensional space-time manifold; this builds up an 11 + 3-dimensional space-time. In this system, firstly, some fields living in the bulk join the fields that live on the 11-dimensional manifold, so that the rank of the gauge fields exceeds the dimension of the algebra; consequently, there emerges an anomaly. To solve this problem, another 11-dimensional manifold is included in the $11+3$-dimensional space-time, and it interacts with the initial manifold by exchanging Chern-Simon fields. This mechanism is able to remove the anomaly. Chern-Simons terms actually produce an extra manifold in the pair of 11-dimensional manifolds of the $11+3$-space-time. Summing up the topology of both the 11-dimensional manifolds and the topology of the exchanged Chern-Simons manifold in the bulk, we conclude that the total topology shrinks to one, which is in agreement with the main idea of the Big Bang theory.
\end{abstract}

\section{Introduction}

Some authors have recently extended general relativity and proposed a Chern-Simons modified gravity in which the Einstein-Hilbert action is supplemented by a parity-violating Chern-Simons term, which couples to gravity via a scalar field. The parity-violating Chern-Simons term is defined as a contraction of the Riemann curvature tensor with its dual and the Chern-Simons scalar field [1]. Ever since, a great deal of contributions and discussions on this particular model has appeared in the literature. For example, the authors of [2] have studied the combined effects of the Lorentz-symmetry violating Chern-Simons and Ricci-Cotton actions for the EinsteinHilbert model in the second-order formalism extended by the inclusion of higher-derivative terms and considered their consequences on the spectrum. In another investigation, the authors have argued about rotating black hole solutions in the $(3+1)$-dimensional Chern-Simons modified gravity by taking account of perturbations around the Schwarzschild solution [3]. They have obtained the zenith-angle dependence of a metric function that corresponds to the frame-dragging effect, by using a constraint equation without choosing the embedding coordinate system. Also, a conserved and symmetric energy-momentum (pseudo)tensor for ChernSimons modified gravity has been built up and it has been shown that the model is Lorentz invariant [4]. In another article, the authors have considered the effect of ChernSimons modified gravity on the quantum phase shift of de Broglie waves in neutron interferometry by applying a unified approach of optical-mechanical analogy in a semiclassical model [5]. In a different scenario, the authors have asserted the consistency of the Gödel-type solutions within the fourdimensional Chern-Simons modified gravity with the nondynamical Chern-Simons coefficient, for various shapes of scalar matter and electromagnetic fields [6]. Finally, in one of the latest versions of the Chern-Simons gravity, the ChernSimons scalar fields are treated as dynamical fields possessing their own stress energy tensor and an evolution equation. This version has been named Dynamical Chern-Simons Modified Gravity (DCSMG) [7-9]. Now, a question arises on what this 
tensor is and what would be the origin of these Chern-Simons terms. We shall here show that our universe is a part of an 11-dimensional manifold which is connected with another 11dimensional manifold by an extra 3-dimensional space. The 11-dimensional manifolds interact with one another via the exchange of Chern-Simons fields which move along the 3dimensional manifold.

Our model is, in fact, a generalization of Kaluza-Klein theory to $11+3$-dimensional space-time. Until now, many discussions have been done on this subject. For example, in one paper some very interesting features of the large $D$ expansion of a Kaluza-Klein theory in $4+D$ dimensions have been considered. This model exhibits a nontrivial large $D$ scaling: in particular, it has been found that the fourdimensional effective cosmological $\Lambda$ constant is of order $1 / D$ [10]. In other researches, the properties of different types of black holes in $D$-dimensional Kaluza-Klein theory have been considered. It is observed that, by reducing dimensions to four, these black holes achieve the same properties of normal black holes in 4-dimensional gravity [11-13].

The main reason for considering higher dimensional world is responding to some main questions and removing some puzzles in field theory and cosmology. For example, what is the reason for the emergence of the difference between fermions and bosons? What is the origin of the emergence of extra terms in generalized uncertainty principles in 4dimensional field theory? What is the origin of the emergence of very big energy at the Big Bang? In 10-dimensional string theory, some of these questions have had a response; however this theory had some anomalies. In 1995, by generalizations of the number of dimensions to 11 , the anomalies in 10dimensional string theory have been removed [14]. However, this theory contains two stable objects like 3-dimensional M2-brane and 6-dimensional M5-brane and designing a 4 -dimensional universe is very hard. Also, this $M$-theory includes some other anomalies which can be removed in theories with more than 11 dimensions like $G$-theory in 14 dimensions [15].

Recently, a new theory has been proposed in 14dimensional space-time that responds to many questions, removes the anomalies in 11-dimensional $M$-theory, and considers the evolution of universe from nothing to present stage. In this theory, at the beginning, two types of G0branes, one with positive energy and one with negative energy, are created from nothing in fourteen dimensions. Then, these branes are compacted on three circles via two different ways (symmetrically and antisymmetrically), and two bosonic and fermionic parts of action for M0-branes are created. By joining $M 0$-branes, supersymmetric $M p$ branes are produced which include the equal number of degrees of freedom for fermions and bosons. Our universe is built on one of $M p$-branes and other $M p$-brane and extra energy play the role of bulk. By dissolving extra energy which is created by compacting actions of $G p$-branes, into our universe, the number of degrees of freedom on it and also its scale factor increase and universe expands. We test $G$-theory with observations and find that the magnitude of the slow-roll parameters and the tensor-to-scalar ratio in this model are a lot smaller than one which are in agreement with predictions of experimental data. Finally, we consider the origin of the extended theories of gravity in $G$-theory and show that these theories could be anomaly-free. And, finally, one of the main results of an extension to 14 dimensions yields the predicted terms in the generalized uncertainty principle [16]. This theory gives the exact form of GUP and explains the reason for the birth of extra terms and their growing in this principle. In this paper, we will show that the physics of 11-dimensional spacing manifold + 3-dimensional algebraic manifold is equal to the physics of 14-dimensional manifold. This helps us to understand why $M$-theory with Lie-three algebra is a true theory and solve many problems in physics. In fact, $M$-theory with Lie-three algebra lives on 14 dimensional manifold where a 3-dimensional part of it corresponds to Lie-3-algebra and the other 11-dimensional part is related to space-time. Also, we show that $M$-theory on 11-dimensional manifold could be the anomaly-free if a three-dimensional algebraic manifold is added to it or its spacial time is increased to 14 dimensions. This 14-dimensional manifold can be broken to two parallel 11-dimensional manifolds which are connected by a three-dimensional Chern-Simons manifold.

In our model, there is a 14-dimensional manifold which can be divided into smaller parts. Each of these parts can form a new smaller manifold. In Horava-Witten mechanism, most of anomalies are removed on an 11-dimensional manifold. We will show that there are more anomalies that may be removed in a new system which is constructed of two parallel 11-dimensional manifolds which are connected by a threedimensional manifold. This system can be created in a 14dimensional space-time. In fact, this system is similar to Bion in string theory. Bion is a system which has been constructed of two branes which are connected by a wormhole. Now, our new Bion has been constructed of two 11-dimensional manifolds which have been connected by a three-dimensional manifold. This new Bion can be a part of a 14-dimensional manifold.

In this model, we will use a generalization of the concept of Lie algebra to an $n$-array bracket. This algebra gives us this opportunity to produce all types of gauge fields by using the relation between brackets and derivatives with respect to strings. In $M$-theory, Lie-three algebra has been used which include 3-dimensional brackets (brackets with 3 arrays). We will show that there is a direct relation between dimensions of brackets and dimensions of manifold and by increasing the number of dimensions of manifold, the number of arrays (number of dimensions) of algebra is increased. To remove anomalies, we have to increase number of dimensions of manifolds. Consequently, the number of dimensions of brackets (number of arrays) should be increased.

Maybe, this question arises: can all anomalies be removed in 10-dimensional superstring theory? In this theory, anomalies depend on the difference between numbers of degrees of freedoms of fermions and bosons. If the number of degrees of freedoms of fermions is equal to the number of degrees of freedoms of bosons, all anomalies can be removed. However, Horava and Witten show that some anomalies appeared due to axial fields and also interactions between fermions that can be removed by extending dimensions to eleven [14,17]. In this paper, it is shown that 11-dimensional theory also has some 
anomalies that can be removed by extending dimensions to 14. Also, we will show that there is a direct relation between number of dimensions and algebra and also by choosing a suitable algebra, anomalies can be removed completely.

Our paper is organized according to the following outline: in Section 2, we devote efforts to show that, by adding up a 3-dimensional manifold to eleven-dimensional gravity, there emerges a Chern-Simons modified gravity. Next, in Section 3, we shall show that if the fields obey a special algebra, Chern-Simons modified gravity is shown to be anomalyfree. However, by increasing the rank of the fields, other anomalies show up. In Section 4, we focus on the removal of the anomaly of this type of gravity in a system composed of two 11-dimensional spaces and a Chern-Simons manifold that connects them. In the last section, we cast a summary and our final considerations.

\section{Chern-Simons Modified Gravity on an 11 + 3-Dimensional Manifold}

We start off by introducing the action of the Dynamical Chern-Simons modified gravity [7-9]:

$$
\begin{aligned}
S_{\mathrm{DCSMG}} & =S_{\mathrm{EH}}+S_{\mathrm{CS}}+S_{\phi}+S_{\mathrm{mat}} \\
S_{\mathrm{EH}} & =\int d^{4} x \sqrt{-g} R \\
S_{\mathrm{CS}} & =\int d^{4} x \sqrt{-g}\left(\frac{1}{2} \epsilon^{\alpha \beta \mu \nu} \phi R_{\alpha \beta \gamma \delta} R_{\mu \nu}^{\gamma \delta}\right) \\
S_{\phi} & =-\int d^{4} x \sqrt{-g}\left[g^{\mu \nu} \partial_{\mu} \phi \partial_{\nu} \phi+2 V(\phi)\right],
\end{aligned}
$$

where $R$ is the curvature and $\phi$ is the Chern-Simons scalar field.

Now, we are going to show that Chern-Simons modified gravity can be obtained from a supergravity which lives on an $11+3$-dimensional manifold. Actually, we assume that our four-dimensional universe is a part of an 11-dimensional manifold that interacts with the bulk in an 11+3-dimensional space-time by exchanging Chern-Simons fields. For this, our departure point is the purely bosonic sector of elevendimensional supergravity and we show that, by adding up a three-dimensional manifold, Chern-Simons terms will appear.

The bosonic piece of the action for a gravity which lives on an eleven-dimensional manifold is given by $[14,17]$

$$
\begin{aligned}
& S_{\text {Bosonic-SUGRA }} \\
& =\frac{1}{\bar{\kappa}^{2}} \int d^{11} x \sqrt{g}\left(-\frac{1}{2} R-\frac{1}{48} G_{I J K L} G^{I J K L}\right)+S_{C G G} \\
& S_{C G G}=-\frac{\sqrt{2}}{3456 \bar{\kappa}^{2}} \int_{M^{11}} d^{11} x \varepsilon^{I_{1} I_{2} \cdots I_{11}} C_{I_{1} I_{2} I_{3}} G_{I_{4} \cdots I_{7}} G_{I_{8} \cdots I_{11}},
\end{aligned}
$$

where the curvature $(R)$ and $G_{I J K L}$ and $C_{I_{1} I_{2} I_{3}}$, given in terms of the gauge field, $A$, and its field-strength, $F$, are cast in what follows [17]:

$$
G_{I J K L}=-\frac{3}{\sqrt{2}} \frac{\kappa^{2}}{\lambda^{2}} \varepsilon\left(x^{11}\right)\left(F_{I J} F_{K L}-R_{I J} R_{K L}\right)+\cdots
$$

$$
\begin{aligned}
\delta C_{A B C}= & -\frac{\kappa^{2}}{6 \sqrt{2} \lambda^{2}} \delta\left(x^{11}\right) \operatorname{tr}\left(\epsilon_{C} F_{A B}-\epsilon_{C} R_{A B}\right) \\
G_{11 A B C}= & \left(\partial_{11} C_{A B C} \pm 23 \text { permutations }\right) \\
& +\frac{\kappa^{2}}{\sqrt{2} \lambda^{2}} \delta\left(x^{11}\right) \omega_{A B C} \\
\delta \omega_{\mathrm{ABC}}= & \partial_{A}\left(\operatorname{tr} \epsilon F_{B C}\right) \\
& +\operatorname{cyclic} \text { permutations of } A, B, C \\
F^{I J}= & \partial^{I} A^{J}-\partial^{J} A^{I} \\
R_{I J}= & \partial_{I} \Gamma_{J \beta}^{\beta}-\partial_{J} \Gamma_{I \beta}^{\beta}+\Gamma_{J \beta}^{\alpha} \Gamma_{I \alpha}^{\beta}-\Gamma_{I \beta}^{\alpha} \Gamma_{J \alpha}^{\beta} \\
\Gamma_{I J K}= & \partial_{I} g_{J K}+\partial_{K} g_{I J}-\partial_{J} g_{I K} \\
G_{I J}= & R_{I J}-\frac{1}{2} R g_{I J} .
\end{aligned}
$$

Here, $\varepsilon\left(x^{11}\right)$ is 1 for $x^{11}>0$ and 1 for $x^{11}<0$ and $\delta\left(x^{11}\right)=\partial \varepsilon / \partial x^{11}$. Both capitalized Latin (e.g., $\left.I, J\right)$ and Greek (e.g., $\beta$ ) indices act on the same manifold and we have only exhibited the free indices $I, J, K$ and the dummy ones $(\alpha, \beta)$. $\epsilon_{C}$ is used as a vector in direction of $C$. This helps the equation that becomes balanced from the indices point of view. The gauge variation of the CGG-action gives the following result [17]:

$$
\begin{aligned}
& \left.\delta S_{C G G}\right|_{11} \\
& \quad=-\frac{\sqrt{2}}{3456 \bar{\kappa}^{2}} \int_{M^{11}} d^{11} x \varepsilon^{I_{1} I_{2} \cdots I_{11}} \delta C_{I_{1} I_{2} I_{3}} G_{I_{4} \cdots I_{7}} G_{I_{8} \cdots I_{11}} \\
& \approx-\frac{\bar{\kappa}^{4}}{128 \lambda^{6}} \int_{M^{10}} \sum_{n=1}^{5}\left(\operatorname{tr} F^{n}-\operatorname{tr} R^{n}+\operatorname{tr}\left(F^{n} R^{5-n}\right)\right),
\end{aligned}
$$

where $\operatorname{tr} F^{n}=\operatorname{tr}\left(F_{\left[I_{1} I_{2}\right.} \cdots F_{\left.I_{2 n-1} I_{2 n}\right]}\right)=$ $\epsilon^{I_{1} I_{2} \cdots I_{2 n-1} I_{2 n}} \operatorname{tr}\left(F_{I_{1} I_{2}} \cdots F_{I_{2 n-1} I_{2 n}}\right) \quad$ and $\operatorname{tr} R^{n}=$ $\operatorname{tr}\left(R_{\left[I_{1} I_{2}\right.} \cdots R_{\left.I_{2 n-1} I_{2 n}\right]}\right)=\epsilon^{I_{1} I_{2} \cdots I_{2 n-1} I_{2 n}} \operatorname{tr}\left(R_{I_{1} I_{2}} \cdots R_{I_{2 n-1} I_{2 n}}\right)$. These terms above cancel the anomaly of $\left(S_{\text {Bosonic-SUGRA }}\right)$ in eleven-dimensional manifold [17]:

$$
\left.\delta S_{\text {CGG }}\right|_{11}=-\delta S_{\text {Bosonic-SUGRA }}=-\delta S_{\text {Bosonic-SUGRA }}^{\text {anomaly }} .
$$

Thus, $S_{C G G}$ is necessary for the anomaly cancelation; so, let us now go on and try to find a good rationale for it. Also, we shall answer the question related to the origin of CGG terms in 11-dimensional supergravity. We actually propose a scenario in which the $C G G$ terms appear in the supergravity action in a way that we do not add them up by hand. To this end, we choose a unified shape for all fields by using the Nambu-Poisson brackets and the properties of string fields $(X)$. We define $[15,18,19]$

$$
I_{J}=\epsilon_{J} I=\epsilon_{J} \frac{\epsilon_{\alpha J}^{\alpha}+\Gamma_{\alpha J}^{\alpha}}{\epsilon_{\alpha J}^{\alpha}+\Gamma_{\alpha J}^{\alpha}}
$$




$$
\begin{gathered}
X^{I_{i}}=y^{I_{i}}+A^{I_{i}}+\epsilon^{I_{i}} \phi-\epsilon^{I_{i} J} \Gamma_{\alpha J}^{\alpha} \\
-\epsilon^{I_{i} J} \sum_{n=1}^{\infty}\left(\Gamma_{\alpha J}^{\alpha}\right)^{-n}+\cdots \\
\left\{X^{I_{i}}, X^{I_{j}}\right\}=F^{I_{i} I_{j}}-R^{I_{i} I_{j}}+\partial^{I_{i}} \phi \partial_{I_{j}} \phi \\
-\frac{1}{2} \epsilon^{I_{i} I_{j} I_{k} I_{m}} \phi R_{I_{i} I_{j} I_{k} I_{m}}+\cdots,
\end{gathered}
$$

where $\phi$ is the Chern-Simons scalar field, $A^{I}$ is the gauge field, $\Gamma$ is related to the curvature $(R)$, and $I$ is a unit vector in the direction of the coordinate which can be expanded in terms of derivatives of metric. In fact, the origin of all matter fields and strings is the same and they are equal to the unit vectors $\left(I_{J}=I \epsilon_{J}\right)$ in addition to some fields $\left(\phi, A^{I}\right)$ which appear as fluctuations of space. The latter may emerge by the interaction of strings which breaks the initial symmetric state. Without string interactions, we have a symmetry that could be explained by a unit vector or a matrix. We can first say that, in the static state, all strings are equal to a unit vector or a matrix and, then, these strings interact with one another, so that the symmetry is broken and fields emerge. Also, $\epsilon^{I_{i} I_{j} I_{k} I_{m}}$ is an antisymmetric tensor that has been attached to antisymmetric curvature and makes a symmetric part. This tensor causes that different states of curvature be regarded. Maybe, this question arises: is $X$ used only for strings in 26dimensional string theory? In fact, this could be a sign for bosonic strings in any dimension and is not related to 26 or 10 dimensions. Using four-dimensional brackets instead of twodimensional ones, we obtain the shape of the GG-terms in supergravity as functions of strings $(X)$ :

$$
\begin{gathered}
G^{I J K L}=\left\{X^{I}, X^{J}, X^{K}, X^{L}\right\}=\sum_{I^{\prime} J^{\prime} K^{\prime} L^{\prime}} \epsilon^{I^{\prime} J^{\prime} K^{\prime} L^{\prime}} \frac{\partial X^{I}}{\partial y^{\prime}} \frac{\partial X^{J}}{\partial y^{J^{\prime}}} \frac{\partial X^{K}}{\partial y^{K^{\prime}}} \frac{\partial X^{L}}{\partial y^{L^{\prime}}} \Longrightarrow \\
\int d^{11} x \sqrt{g}\left(G_{I J K L} G^{I J K L}\right)=\int d^{11} x \sqrt{g}\left(\sum_{I J K L} \epsilon_{I^{\prime} J^{\prime} K^{\prime} L^{\prime}} \frac{\partial X_{I}}{\partial y_{I^{\prime}}} \frac{\partial X_{J}}{\partial y_{J^{\prime}}} \frac{\partial X_{K}}{\partial y_{K^{\prime}}} \frac{\partial X_{L}}{\left.\partial y_{L^{\prime}} \sum_{I^{\prime} J^{\prime} K^{\prime} L^{\prime}} \epsilon^{I^{\prime} J^{\prime} K^{\prime} L^{\prime}} \frac{\partial X^{I}}{\partial y^{I^{\prime}}} \frac{\partial X^{J}}{\partial y^{J^{\prime}}} \frac{\partial X^{K}}{\partial y^{K^{\prime}}} \frac{\partial X^{L}}{\partial y^{L^{\prime}}}\right) .}\right.
\end{gathered}
$$

The equation above helps us to extract the CGG terms from the $G G$-terms in supergravity. To this end, we must add a three-dimensional manifold (related to a Lie-three-algebra) to eleven-dimensional supergravity by using the properties of strings $(X)$ in Nambu-Poisson brackets [15]:

$$
\begin{aligned}
X^{I_{i}} & =y^{I_{i}}+A^{I_{i}}-\epsilon^{I_{i} J} \Gamma_{\alpha J}^{\alpha}-\epsilon^{I_{i} J} \sum_{n=1}^{\infty}\left(\Gamma_{\alpha J}^{\alpha}\right)^{-n} \Longrightarrow \\
\frac{\partial X^{I_{5}}}{\partial y^{I_{5}}} & \approx \delta\left(y^{I_{5}}\right)+\cdots \\
\frac{\partial X^{I_{6}}}{\partial y^{I_{6}}} & \approx \delta\left(y^{I_{6}}\right)+\cdots \\
\frac{\partial X^{I_{7}}}{\partial y^{I_{7}}} & \approx \delta\left(y^{I_{7}}\right)+\cdots \\
\int_{M^{N=3}} & \longrightarrow \int_{y^{I_{5}}+y^{I_{6}}+y^{I_{7}}} \epsilon^{I_{5}^{\prime} I_{6}^{\prime} I_{7}^{\prime}} \frac{\partial X^{I_{5}}}{\partial y^{I_{5}^{\prime}}} \frac{\partial X^{I_{6}}}{\partial y^{I_{6}^{\prime}}} \frac{\partial X^{I_{7}}}{\partial y^{I_{7}^{\prime}}}=1+\cdots
\end{aligned}
$$

where the integration has been carried out over a threedimensional manifold with coordinates $\left(y^{I_{5}}, y^{I_{6}}, y^{I_{7}}\right)$ and, consequently, the integration can be done by using that $\left.\int_{y^{I_{5}}+y^{I_{6}}+y^{I_{7}}}=\int d y^{I_{5}} \int d y^{I_{6}} \int d y^{I_{7}}\right)$. The result above shows that, by ignoring fluctuations of space which yield production of fields, the area of each three-dimensional manifold can shrink to one and the result of the integration over that manifold goes to one. When we add one manifold to the other, the integration will be the product of an integration over each manifold, for the coordinates of the added manifolds increase the elements of integration. By adding the three-dimensional manifold of (8) to the eleven-dimensional manifold of (7), we get

$$
\begin{aligned}
& \int_{M^{N=3}} \times \int_{M^{11}} \sqrt{g}\left(G_{I_{1} I_{2} I_{3} I_{4}} G^{I_{1} I_{2} I_{3} I_{4}}\right) \\
& =\int_{M^{11}+y^{I_{5}}+y^{I_{6}}+y^{I_{7}}} \sqrt{g} \epsilon^{I_{5}^{\prime} I_{6}^{\prime} I_{7}^{\prime}} G_{I_{1} I_{2} I_{3} I_{4}} G^{I_{1} I_{2} I_{3} I_{4}} \frac{\partial X^{I_{5}}}{\partial y^{I_{5}^{\prime}}} \frac{\partial X^{I_{6}}}{\partial y^{I_{6}^{\prime}}} \frac{\partial X^{I_{7}}}{\partial y^{I_{7}^{\prime}}} \\
& =\int_{M^{11}+M^{N=3}} \sqrt{g} C G G \Longrightarrow \\
& C^{I_{5} I_{6} I_{7}}=\sum_{I_{5}^{\prime} I_{6}^{\prime} I_{7}^{\prime}} \epsilon^{I_{5}^{\prime} I_{6}^{\prime} I_{7}^{\prime}} \frac{\partial X^{I_{5}}}{\partial y^{I_{5}^{\prime}}} \frac{\partial X^{I_{6}}}{\partial y^{I_{6}^{\prime}}} \frac{\partial X^{I_{7}}}{\partial y_{7}^{I_{7}^{\prime}}} .
\end{aligned}
$$

This equation presents three results we should comment on: (1) CGG terms may appear in the action of supergravity by adding a three-dimensional manifold, related to the Lie-three-algebra added to eleven-dimensional supergravity. (2) 11-dimensional manifold + three-Lie algebra $=14$ dimensional supergravity. (3) The shape of the $C$-terms is now clear in terms of the string fields, $\left(X^{i}\right)$.

Substituting (6), (7), and (8) into (9) yields

$$
\begin{aligned}
& \int_{M^{11}+M^{N=3}} \sqrt{g} C G G \\
& =\int_{M^{11}+M^{N=3}} \sqrt{g}\left(\frac{1}{2} \epsilon^{I_{i} I_{j} I_{k} I_{m}} \phi R_{I_{k} I_{m} I_{l} I_{n}} R_{I_{i} I_{j} I_{n}}^{I}-\partial_{I_{i}} \phi \partial^{I_{j}} \phi\right) \\
& \quad-\frac{1}{2} \int_{M^{11}+M^{N=3}} \sqrt{g}\left(\phi \epsilon_{I_{k} I_{m}}^{I_{i} I_{j}} F^{I_{k} I_{m}} F_{I_{i} I_{j}}\right)+\cdots
\end{aligned}
$$


In the equation above, the first integration is in agreement with previous predictions of Chern-Simons gravity in [79] and can be reduced to the four-dimensional ChernSimons modified gravity of (1). Also, the second integration is related to the interaction of gauge fields with Chern-Simons fields. Thus, this model not only produces the Chern-Simons modified gravity, but also exhibits some modifications to it. Still, these results show that our universe is a part of oneeleven-dimensional manifold which interacts with a bulk in a 14-dimensional space-time by exchanging Chern-Simons scalars.

\section{Anomalies in Chern-Simons Modified Gravity}

In this section, we shall consider various anomalies which may be induced in Chern-Simons modified gravity. Although we expect that terms in the gauge variation of the ChernSimons action remove the anomaly in eleven-dimensional supergravity, we will observe that some extra anomalies are produced by the Chern-Simons field. It is our goal to show that these anomalies depend on the algebra and thus, by choosing a suitable algebra in this model, all anomalies can be removed. To obtain the anomalies of the Chern-Simons theory, we should reobtain the gauge variation of the CGGaction in (4) in terms of field-strengths and curvatures. To this end, by using (8) and (9), we can work out the gauge variation of $C[15]$ :

$$
\begin{aligned}
& X^{I_{i}}=y^{I_{i}}+A^{I_{i}}-\epsilon^{I_{i} J} \Gamma_{\alpha J}^{\alpha}-\epsilon^{I_{i} J} \sum_{n=1}^{\infty}\left(\Gamma_{\alpha J}^{\alpha}\right)^{-n} \Longrightarrow \\
& \frac{\partial \delta_{A} X^{I}}{\partial y^{I}}=\delta\left(y^{I}\right) \Longrightarrow \\
& \int_{M^{N=3}+M^{11}} \delta_{A} C^{I_{5} I_{6} I_{7}} \\
& =\int_{M^{N=3}+M^{11}} \sum_{I_{5}^{\prime} I_{6}^{\prime} I_{7}^{\prime}} \epsilon^{I_{5}^{\prime} I_{6}^{\prime} I_{7}^{\prime}} \delta_{A}\left(\frac{\partial X^{I_{5}}}{\partial y^{I_{5}^{\prime}}} \frac{\partial X^{I_{6}}}{\partial y^{I_{6}^{\prime}}} \frac{\partial X^{I_{7}}}{\partial y^{I_{7}^{\prime}}}\right)= \\
& \int_{M^{N=3}+M^{10}} \sum_{I_{5}^{\prime} I_{6}^{\prime}} \epsilon^{I_{5}^{\prime} I_{6}^{\prime}}\left(\frac{\partial X^{I_{5}}}{\partial y^{I_{5}^{\prime}}} \frac{\partial X^{I_{6}}}{\partial y^{I_{6}^{\prime}}}\right)=\int_{M^{N=3}+M^{10}}\left(F^{I_{i} I_{j}}\right. \\
& \left.-R^{I_{i} I_{j}}+\partial^{I_{i}} \phi \partial_{I_{j}} \phi-\frac{1}{2} \epsilon^{I_{i} I_{j} I_{k} I_{m}} \phi R_{I_{i} I_{j} I_{k} I_{m}}+\cdots\right)
\end{aligned}
$$

Using the equation above and (7), we get the gauge variation of the CGG action given in (9):

$$
\begin{aligned}
\delta & \int_{M^{11}+M^{N=3}} \sqrt{g} C G G \\
& =\int_{M^{10}+M^{N=3}} \sqrt{g} \sum_{n=1}^{5}\left(\operatorname{tr} F^{n}-\operatorname{tr} R^{n}+\operatorname{tr}\left(F^{n} R^{5-n}\right)\right) \\
& +\int_{M^{10}+M^{N=3}} \sqrt{g}\left(\sum_{n=1}^{5}\left(\operatorname{tr}\left(F^{n}\left(\partial^{I_{i}} \phi \partial_{I_{j}} \phi\right)^{5-n}\right)\right)+\operatorname{tr}\left(F^{n}\left(\epsilon^{I_{i} I_{j} I_{k} I_{m}} R_{I_{i} I_{j} I_{k} I_{m}} \phi\right)^{5-n}\right)\right) \\
& -\int_{M^{10}+M^{N=3}} \sqrt{g}\left(\sum_{n=1}^{5}\left(\operatorname{tr}\left(R^{n}\left(\partial^{I_{i}} \phi \partial_{I_{j}} \phi\right)^{5-n}\right)\right)+\operatorname{tr}\left(R^{n}\left(\epsilon^{I_{i} I_{j} I_{k} I_{m}} R_{I_{i} I_{j} I_{k} I_{m}} \phi\right)^{5-n}\right)\right)+\cdots .
\end{aligned}
$$

The first line of this equation removes the anomaly on the 11dimensional manifold of (4); however, the second and third lines show that extra anomalies can emerge due to the ChernSimons fields. We can show that if we choose a suitable algebra for the 11-dimensional manifold, all anomalies can be swept out. We can extend our discussion to a $D$-dimensional manifold with a Lie- $N$-algebra. In fact, we wish to obtain a method that makes all supergravities, with arbitrary dimension, anomaly-free. To this end, we make use of the properties of Nambu-Poisson brackets and strings $(X)$ in (6) to obtain a unified definition for different terms in supergravity and rewrite action (4) as follows:

$$
\begin{aligned}
& \delta S_{C G G}=-\frac{\bar{\kappa}^{4}}{128 \lambda^{6}} \int_{M^{10}} \epsilon^{I_{1} I_{2} \cdots I_{10}}\left\{X^{I_{1}}, X^{I_{2}}\right\}\left\{X^{I_{3}}, X^{I_{4}}\right\} \\
& \cdot\left\{X^{I_{5}}, X^{I_{6}}\right\}\left\{X^{I_{7}}, X^{I_{8}}\right\}\left\{X^{I_{9}}, X^{I_{10}}\right\} .
\end{aligned}
$$

In the equation above, we only used the Lie-twoalgebra with two-dimensional bracket; however, it is not clear whether this algebra is true. In fact, for $M$-theory, Liethree-algebra with three-dimensional bracket $[18,19]$ is more suitable. To obtain the exact form of the Lie algebra which is suitable for $D$-dimensional space-time, we shall generalize the dimension of space-time from eleven to $D$ and the algebra from two to $N$ and use the following Nambu-Poisson brackets [19]:

$$
\begin{gathered}
\int_{M^{D}}\left\{X^{I_{i}}, X^{I_{j}}\right\} \cdots \longrightarrow \\
\int_{M^{N+D}} \epsilon_{J_{1} J_{2} \cdots J_{N}}^{I_{i} I_{j}}\left\{X^{J_{1}}, X^{J_{2}}, \ldots, X^{J_{N}}\right\} \cdots .
\end{gathered}
$$

In this equation, we have added a new manifold, related to the algebra, to the world manifold. In fact, we have to 
regard both algebraic $\left(M^{N}\right)$ and space-time $\left(M^{D}\right)$ manifolds to achieve the exact results. For the $N$-dimensional algebra, we introduce the following fields:

$$
\begin{aligned}
& X^{J_{N}} \longrightarrow y^{J_{N}}+\epsilon_{J_{1}, J_{2}, \ldots, J_{N-1}}^{J_{N}} A^{J_{1}, J_{2}, \ldots, J_{N-1}} \\
& -\epsilon_{J_{1}, J_{2}, \ldots, J_{N-1}}^{J_{N}} \partial^{J_{4}} \cdots \partial^{J_{N-1}} \Gamma^{J_{1}, J_{2}, J_{3}} \\
& F^{J_{1} \cdots J_{N}}=\epsilon_{J_{1}, J_{2}, \ldots, J_{N-1}}^{J_{N}} \partial_{J_{N}} A^{J_{1}, J_{2}, \ldots, J_{N-1}} \\
& \partial^{J_{5}} \cdots \partial^{J_{N}} R^{J_{1} \cdots J_{4}}=\epsilon_{J_{1}, J_{2}, \ldots, J_{N-1}}^{J_{N}} \partial_{J_{N}} \partial^{J_{4}} \cdots \partial^{J_{N-1}} \Gamma^{J_{1} J_{2} J_{3}} \\
& +\cdots \text {, }
\end{aligned}
$$

where

$$
\begin{aligned}
\epsilon_{J_{1} J_{2} \cdots J_{N}}^{I_{i} I_{j}} & =\epsilon^{I_{i} I_{j}} \epsilon_{J_{1} J_{2} \cdots J_{N}} \\
\epsilon_{J_{1} J_{2} \cdots J_{N}}^{I_{i}} & =\delta_{\left[J_{1} J_{2} \cdots J_{N}\right]}^{I_{i}} \\
\delta_{\left[J_{1} J_{2} \cdots J_{N}\right]} & =\delta_{J_{1} J_{2} \cdots J_{N}}-\delta_{J_{2} J_{1} \cdots J_{N}}+\cdots .
\end{aligned}
$$

Here, $\delta$ is the generalized Kronecker delta. With definitions in (15), we can obtain the explicit form of the $N$ dimensional Nambu-Poisson brackets in terms of fields:

$$
\begin{aligned}
& \int_{M^{N}+M^{D}}\left\{X^{J_{1}}, X^{J_{2}}, \ldots, X^{J_{N}}\right\} \\
& \quad=\int_{M^{N}} \sum_{J_{1}, J_{2}, \ldots, J_{N}} \epsilon^{J_{1}, J_{2}, \ldots, J_{N}} \frac{\partial X^{J_{1}}}{\partial y^{J_{1}}} \frac{\partial X^{J_{2}}}{\partial y^{J_{2}}} \cdots \frac{\partial X^{J_{N}}}{\partial y^{J_{N}}} \\
& \quad \approx \int_{M^{N}+M^{D}}\left(F^{J_{1} \cdots J_{N}}-\partial^{J_{5}} \cdots \partial^{J_{N}} R^{J_{1} \cdots J_{4}}\right) .
\end{aligned}
$$

Substituting (14), (15), and (17) in (13), which is another form of (4), and replacing 11-dimensional manifold with $D$ dimensional manifold, we obtain

$$
\begin{aligned}
& \left.\delta S_{C G G}\right|_{D+1}=-Z \int_{M^{D}} \epsilon_{I_{1} I_{2} \cdots I_{D}}\left\{X^{I_{1}}, X^{I_{2}}\right\}\left\{X^{I_{3}}, X^{I_{4}}\right\} \\
& \cdots\left\{X^{I_{D-1}}, X^{I_{D}}\right\} \\
& =-Z \int_{M^{D+N}} \epsilon_{I_{1} I_{2} \cdots I_{D}} \epsilon_{J_{1}^{1} J_{2}^{1} \cdots J_{N}^{1}}^{I_{1} I_{2}}\left\{X^{J_{1}^{1}}, X^{J_{2}^{1}}, \ldots, X^{J_{N}^{1}}\right\} \\
& \cdot \epsilon_{J_{1}^{2} J_{2}^{2} \cdots J_{N}^{2}}^{I_{3} I_{4}}\left\{X^{J_{1}^{2}}, X^{J_{2}^{2}}, \ldots, X^{J_{N}^{2}}\right\} \\
& \cdots \epsilon_{J_{1}^{D / 2} J_{2}^{D / 2} \ldots J_{N}^{D / 2}}^{I_{D-1} I_{D}}\left\{X^{J_{1}^{D / 2}}, X^{J_{2}^{D / 2}}, \ldots, X^{J_{N}^{D / 2}}\right\} \\
& =-Z \int_{M^{D+N}} \epsilon_{I_{1} I_{2} \cdots I_{D}} \epsilon_{J_{1}^{1} J_{2}^{1} \cdots J_{N}^{1}}^{I_{1} I_{2}} \epsilon_{J_{1}^{2} I_{2}^{2} \cdots J_{N}^{2}}^{I_{3} I_{4}} \\
& \cdots \epsilon_{J_{1}^{D / 2} J_{2}^{D / 2} \cdots J_{N}^{D / 2}}^{I_{D-1} I_{D}}\left(F^{J_{1}^{1} \cdots J_{N}^{1}}-\partial^{J_{5}^{1}} \cdots \partial^{J_{N}^{1}} R^{J_{1}^{1} \cdots J_{4}^{1}}\right) \\
& \times\left(F^{J_{1}^{2} \cdots J_{N}^{2}}-\partial^{J_{5}^{2}} \cdots \partial^{J_{N}^{2}} R^{J_{1}^{2} \cdots J_{4}^{2}}\right) \cdots\left(F^{J_{D / 2}^{1} \cdots J_{N}^{D / 2}}-\partial^{J_{5}^{D / 2}}\right. \\
& \left.\cdots \partial^{J_{N}^{D / 2}} R^{J_{1}^{D / 2} \cdots J_{4}^{D / 2}}\right)
\end{aligned}
$$

where $Z$ is a constant related to the algebra. This equation shows that the gauge variation of the action depends on the rank- $N$ field-strength. The action above is not actually directly zero, and there emerges an anomaly. Now, we use properties of $\epsilon$ and rewrite (18) as below:

$$
\begin{aligned}
& \left.\delta S_{C G G}\right|_{D+1}=-Z \int_{M^{D+N}} W(D, N) \\
& \cdot \epsilon^{\chi_{1} \chi_{2} \cdots \chi_{D / 2}}\left(F^{\chi_{1}}-\partial^{J_{5}^{1}} \cdots \partial^{\chi_{1}-4} R^{J_{1}^{1} \cdots J_{4}^{1}}\right) \times\left(F^{\chi_{2}}\right. \\
& \left.\quad-\partial^{J_{5}^{2}} \cdots \partial^{\chi_{2}-4} R^{J_{1}^{2} \cdots J_{4}^{2}}\right) \cdots\left(F^{\chi_{D / 2}}\right. \\
& \left.\quad-\partial^{J_{5}^{D / 2}} \cdots \partial^{\chi_{D / 2}-4} R^{J_{1}^{D / 2} \cdots J_{4}^{D / 2}}\right) .
\end{aligned}
$$

In (19), $\chi, \epsilon^{\chi_{1} \chi_{2} \cdots \chi_{D / 2}}$ and $W(D, N)$ can be obtained as

$$
\begin{aligned}
\chi_{i} & =J_{1}^{i} \cdots J_{N}^{i} \\
\epsilon^{I_{1} I_{2} \cdots I_{D}} \epsilon_{J_{1}^{1} J_{2}^{1} \cdots J_{N}^{1}}^{I_{1} I_{2}} \epsilon_{J_{1}^{2} J_{2}^{2} \cdots J_{N}^{2}}^{I_{3} I_{4}} \cdots \epsilon_{J_{1}^{D-1} J_{2}^{D / 2} \cdots J_{N}^{D / 2}}^{I_{D-1} I_{D}} & \epsilon^{I_{1} I_{2} \cdots I_{D}} \epsilon^{I_{1} I_{2}} \epsilon_{J_{1}^{1} J_{2}^{1} \cdots J_{N}^{1}} \epsilon^{I_{3} I_{4}} \epsilon_{J_{1}^{2} J_{2}^{2} \cdots J_{N}^{2}} \\
\cdots & \epsilon^{I_{D-1} I_{D}} \epsilon_{J_{1}^{D / 2} J_{2}^{D / 2} \cdots J_{N}^{D / 2}}=W(D, N) \epsilon^{\chi_{1} \chi_{2} \cdots \chi_{D / 2}} \\
W & (D, N)=\left(\left[\frac{(D+2)(D-2)}{8}-N\left(\frac{D}{2}-1\right)\right]\right. \\
& {\left.\left[\frac{(D+2)(D-2)}{8}-1-N\left(\frac{D}{2}-1\right)\right] \cdots 1\right) } \\
& \cdot\left(\left[N\left(\frac{D}{2}-1\right)\right]\left[N\left(\frac{D}{2}-1\right)-1\right] \cdots 1\right) U(\delta),
\end{aligned}
$$

where $U$ is a function of the generalized Kronecker delta. On the other hand, $\left.\delta S_{C G G}\right|_{D+1}$ has been added to the main action of supergravity to remove its anomaly. Thus, we can write

$$
\begin{aligned}
& \left.\delta S_{C G G}\right|_{D+1}=-\left.\delta S_{\text {Bosonic-SUGRA }}\right|_{D+1} \\
& \quad=-\left.S_{\text {Bosonic-SUGRA }}^{\text {anomaly }}\right|_{D+1}=0 \Longrightarrow \\
& W(D, N)=\left(\left[\frac{(D+2)(D-2)}{8}-N\left(\frac{D}{2}-1\right)\right]\right. \\
& \left.\cdot\left[\frac{(D+2)(D-2)}{8}-1-N\left(\frac{D}{2}-1\right)\right] \cdots 1\right) \\
& \quad \times\left(\left[N\left(\frac{D}{2}-1\right)\right]\left[N\left(\frac{D}{2}-1\right)-1\right] \cdots 1\right) U=0 \Longrightarrow \\
& N \leq \frac{(D+2)(D-2)}{8(D / 2-1)} .
\end{aligned}
$$

This equation indicates that, for a $(D+1)$-dimensional spacetime, the dimension of the Lie algebra should be equal to or less than a critical value. Under these conditions, the ChernSimons gravity is free from anomalies and we do not need an extra manifold. On the other hand, as we show in (15), the dimension of the algebra determines the dimension of the field-strength. This means that, for a Lie- $N$-algebra, fieldstrengths should have at most $N$ indices. For example, for a 
manifold with 11 dimensions, the algebra can be of order three as predicted in recent papers $[18,19]$ and field-strengths may have three indices. In fact, in above equation, we have shown that the physics of an 11-dimensional spacing manifold plus a 3-dimensional algebraic manifold is equal to the physics of 14-dimensional manifold. This helps us to understand why $M$-theory with Lie-three algebra is a true theory and solves many problems in physics. In fact, $M$-theory with Liethree algebra lives on 14-dimensional manifold where a 3dimensional part of it corresponds to Lie-3-algebra and the other 11-dimensional part is related to space-time. Also, we show that $M$-theory on 11-dimensional manifold could be the anomaly-free if a three-dimensional algebraic manifold is added to it or its spacial time is increased to 14 dimensions. This 14-dimensional manifold can be broken to two parallel 11-dimensional manifold which are connected by a threedimensional Chern-Simons manifold.

\section{A Chern-Simons Manifold between Two 11-Dimensional Manifolds in an $11+3$ Dimensional Space-Time}

In the previous section, we have found that, for an elevendimensional manifold, the suitable algebra which removes the anomaly in Chern-Simons gravity is a three-dimensional Lie algebra. This means that the rank of the fields can be of order two or three. However, (2) shows that the rank of the fields may be higher than three in eleven-dimensional supergravity. Thus, in Chern-Simons gravity theory which lives on an eleven-dimensional manifold, some extra anomalies are expected to show up. To remove them, we assume that there is another 11-dimensional manifold in the 14dimensional space-time which interacts with the first one by exchanging Chern-Simons fields. These fields produce a Chern-Simons manifold that connects these two elevendimensional manifolds (see Figure 1.) Thus, in this model, we have two $G G$ terms which live on 11-dimensional manifolds (see (2)) and two CGG terms in the bulk so that each of them interacts with one of the 11-dimensional manifolds.

We can write the supergravity in 14-dimensional spacetime as follows:

$$
\begin{aligned}
& S_{\text {SUGRA-14 }}=\int_{M^{N=3}}\left(\int_{M^{11}} G G+\int_{M^{11}} \bar{C} G \bar{G}\right. \\
& \left.+\int_{M^{11}} C \bar{G} G+\int_{M^{11}} \bar{G} \bar{G}\right)=\left(\int_{M^{14}} C G G\right. \\
& \left.+\int_{M^{14}} \bar{C} G \bar{G}+\int_{M^{14}} C \bar{G} G+\int_{M^{14}} \bar{C} \bar{G} \bar{G}\right) .
\end{aligned}
$$

In this equation, $C G G$ and $\bar{C} \bar{G} \bar{G}$ are related to the ChernSimons gravities which live on the two eleven-dimensional manifolds and are extracted from $G G$ and $\bar{G} \bar{G}$ terms. Also, $\bar{C} G \bar{G}$ and $C \bar{G} G$ correspond to the Chern-Simons fields which are exchanged between the two manifolds in 14-dimensional space-time. By generalizing the results of (3), (6), and (11), we get

$$
G_{I J K L} \sim-\left(F_{I J} F_{K L}-R_{I J} R_{K L}\right)+\partial_{I} \phi \partial_{J} \phi R_{K L} \cdots
$$

$$
\begin{aligned}
& \delta C_{I_{i} I_{j} I_{k}} \sim-\epsilon_{I_{k}} \operatorname{tr}\left(F_{I_{i} I_{j}}-R_{I_{i} I_{j}}+\partial_{I_{i}} \phi \partial_{I_{j}} \phi\right. \\
& \left.-\frac{1}{2} \epsilon^{I_{i} I_{j} I_{k} I_{m}} \phi R_{I_{i} I_{j} I_{k} I_{m}}+\cdots\right) \\
& \bar{G}_{I J K L} \sim-\left(\bar{F}_{I J} \bar{F}_{K L}-\bar{R}_{I J} \bar{R}_{K L}\right)+\partial_{I} \bar{\phi} \partial_{J} \bar{\phi} \bar{R}_{K L} \cdots \\
& \delta C_{I_{i} I_{j} I_{k}} \sim-\epsilon_{I_{k}} \operatorname{tr}\left(\bar{F}_{I_{i} I_{j}}-\bar{R}_{I_{i} I_{j}}+\partial_{I_{i}} \bar{\phi} \partial_{I_{j}} \bar{\phi}\right. \\
& \left.-\frac{1}{2} \epsilon^{I_{i} I_{j} I_{k} I_{m}} \bar{R}_{I_{i} I_{j} I_{k} I_{m}}+\cdots\right) .
\end{aligned}
$$

Here, the F's, $R$ 's, and $\phi$ 's live on one of the supergravity manifolds as depicted in Figure 1, whereas the $\bar{F}$ 's, $\bar{R}$ 's, and $\bar{\phi}$ 's are fields of the other supergravity manifold. To obtain their relations, we should make use of (12) and the gauge variation of the actions (22); in doing so, we obtain

$$
\begin{aligned}
\delta S_{\text {SUGRA-14 }} & =\int_{M^{14}} \sum_{n=1}^{5}\left(\left\{X^{I_{i}}, X^{I_{j}}\right\}+\left\{\bar{X}^{I_{i}}, \bar{X}^{I_{j}}\right\}\right)^{n}=0 \longrightarrow \\
\left\{X^{I_{i}}, X^{I_{j}}\right\} & =-\left\{\bar{X}^{I_{i}}, \bar{X}^{I_{j}}\right\} \longrightarrow \\
X^{I_{i}} & =i \bar{X}^{I_{i}} \longrightarrow \\
y^{I_{i}} & =i \bar{y}^{I_{i}} \\
A^{I_{i}} & =i \bar{A}^{I_{i}} \\
\phi & =i \bar{\phi} .
\end{aligned}
$$

These results show that, to remove the anomaly in 14dimensional space-time, coordinates and fields in one of the eleven-dimensional manifolds should be equal to coordinates and fields in the other manifold in addition to one extra $i$. This implies that time- or space-like coordinates and fields in one manifold transmute into space- or time-like coordinates and fields of another manifold. For example, the zeroth coordinate which is known as time on one manifold will transmute into a space coordinate of the other manifold. If our universe with one time and three space coordinates is located on one of the manifolds, an antiuniverse with one space and three times is located in the other manifold.

Now, we shall show that, by substituting the results of (24) into the action of (22), the topology of the 14-dimensional manifold tends to one. This means that the world with all its matter began from a point and it may be thought of as a signature of Big Bang in our proposal. To this end, using (6), (7), (9), and (12), we rewrite CGG terms in terms of derivatives of scalar strings:

$$
\begin{gathered}
\int_{M^{11}+M^{N=3}} C G G=k \int_{M^{N=3}} \int_{M^{11}}\left(\delta^{11}(y)\right. \\
\left.+\sum_{n=1}^{6}\left(\frac{\partial X^{I}}{\partial y^{I}}\right)^{6-n}\left(\left\{X^{I_{i}}, X^{I_{j}}\right\}\right)^{n}\right),
\end{gathered}
$$




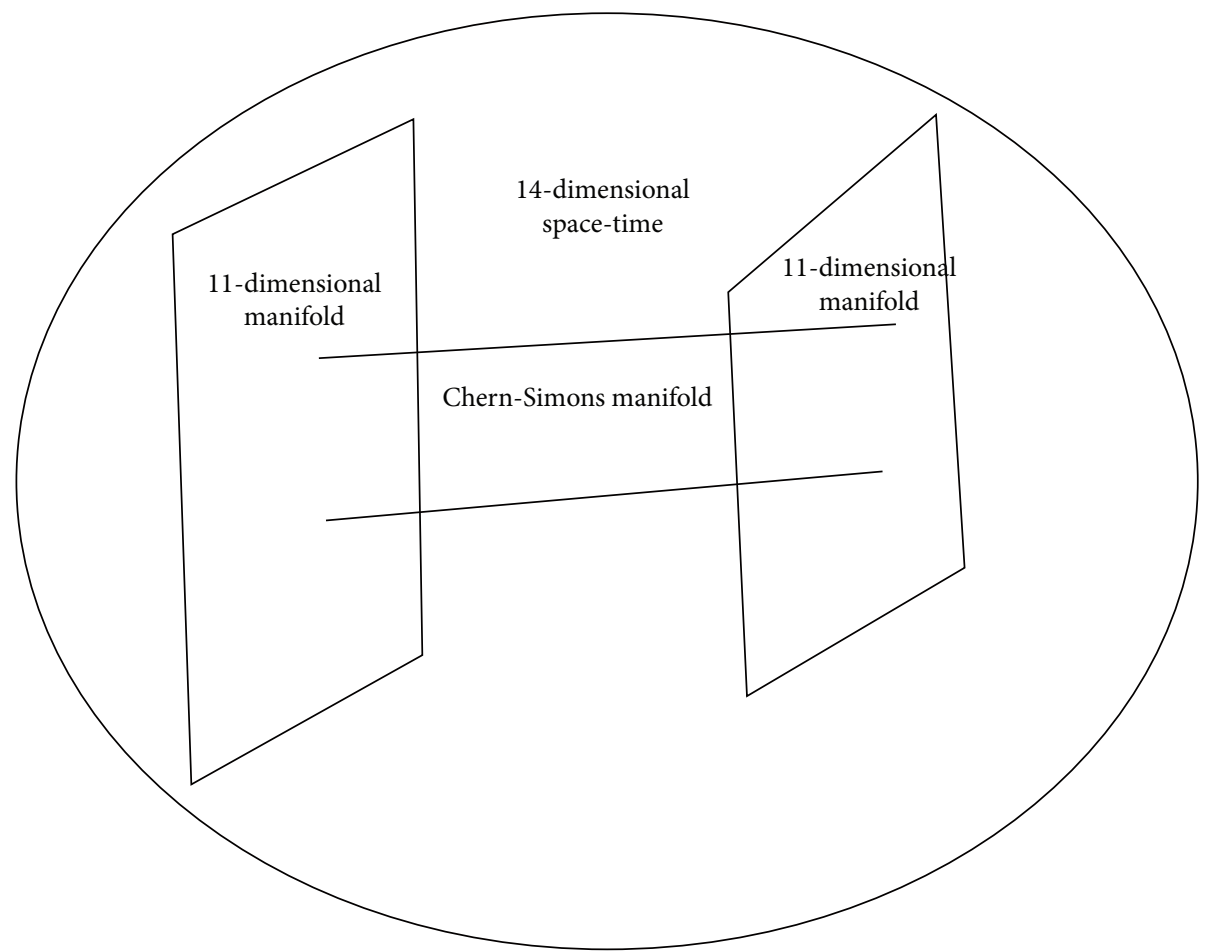

FIGURE 1: Two eleven-dimensional manifolds + Chern-Simons manifold in 14-dimensional space-time.

where $k$ is a constant. There are similar results for other terms in 14-dimensional supergravity:

$$
\begin{aligned}
& \int_{M^{11}+M^{N=3}} \bar{C} \bar{G} \bar{G}=k \int_{M^{N=3}} \int_{M^{11}}\left(\delta^{11}(y)\right. \\
& \left.+\sum_{n=1}^{6}\left(\frac{\partial \bar{X}^{I}}{\partial \bar{y}^{I}}\right)^{6-n}\left(\bar{F}^{n}-\bar{R}^{n}+\cdots\right)\right)=k \int_{M^{N=3}} \int_{M^{11}}\left(\delta^{11}(y)\right. \\
& \left.+\sum_{n=1}^{6}\left(\frac{\partial \bar{X}^{I}}{\partial \bar{y}^{I}}\right)^{6-n}\left(\left\{\bar{X}^{I_{i}}, \bar{X}^{I_{j}}\right\}\right)\right)^{n} \\
& \int_{M^{11}+M^{N=3}} \bar{C} G \bar{G}=k \int_{M^{N=3}} \int_{M^{11}}\left(\delta^{11}(y)\right. \\
& \left.+\sum_{n=1}^{6} \sum_{m=0}^{n}\left(\frac{\partial \bar{X}^{I}}{\partial \bar{y}^{I}}\right)^{6-n}\left(\bar{F}^{m}-\bar{R}^{m}+\cdots\right)\left(F^{n-m}-R^{n-m}+\cdots\right)\right) \\
& \quad k \int_{M^{N=3}} \int_{M^{11}}\left(\delta^{11}(y)\right. \\
& \left.+\sum_{n=1}^{6} \sum_{m=0}^{n}\left(\frac{\partial \bar{X}^{I}}{\partial \bar{y}^{I}}\right)^{6-n}\left(\left\{\bar{X}^{I_{i}}, \bar{X}^{I_{j}}\right\}\right)^{n-m}\left(\left\{X^{I_{i}}, X^{I_{j}}\right\}\right)^{m}\right) \\
& \int_{M^{11}+M^{N=3}} C \bar{G} G=k \int_{M^{N=3}} \int_{M^{11}}\left(\delta^{11}(y)\right. \\
& \left.+\sum_{n=1}^{6} \sum_{m=0}^{n}\left(\frac{\partial \bar{X}^{I}}{\partial \bar{y}^{I}}\right)^{6-n}\left(\bar{F}^{n-m}-\bar{R}^{n-m}+\cdots\right)\left(F^{m}-R^{m}+\cdots\right)\right)
\end{aligned}
$$

$$
\begin{aligned}
& =k \int_{M^{N=3}} \int_{M^{11}}\left(\delta^{11}(y)\right. \\
& \left.+\sum_{n=1}^{6} \sum_{m=0}^{n}\left(\frac{\partial \bar{X}^{I}}{\partial \bar{y}^{I}}\right)^{6-n}\left(\left\{\bar{X}^{I_{i}}, \bar{X}^{I_{j}}\right\}\right)^{m}\left(\left\{\mathrm{X}^{I_{i}}, X^{I_{j}}\right\}\right)^{n-m}\right) .
\end{aligned}
$$

Using the results in (24) and substituting (25) and (26) in (24), we obtain

$$
\begin{aligned}
& \left(\frac{\partial X^{I}}{\partial y^{I}}\right)=\left(\frac{\partial \bar{X}^{I}}{\partial \bar{y}^{I}}\right) \longrightarrow \\
& S_{\text {SUGRA-14 }}=k \int_{M^{N=3}} \int_{M^{11}}\left(\delta^{11}(y)\right. \\
& \left.\quad+\sum_{n=1}^{6}\left(\frac{\partial X^{I}}{\partial y^{I}}\right)^{6-n}\left(\left\{X^{I_{i}}, X^{I_{j}}\right\}+\left\{\bar{X}^{I_{i}}, \bar{X}^{I_{j}}\right\}\right)^{n}\right) .
\end{aligned}
$$

On the other hand, results in (24) show that $\left(\left\{X^{I_{i}}, X^{I_{j}}\right\}=\right.$ $\left.-\left\{\bar{X}^{I_{i}}, \bar{X}^{I_{j}}\right\}\right)$. Thus, we can conclude that the action given above tends to an action on the three-dimensional manifold.

$$
\begin{gathered}
\left\{X^{I_{i}}, X^{I_{j}}\right\}=-\left\{\bar{X}^{I_{i}}, \bar{X}^{I_{j}}\right\} \\
S_{\text {SUGRA-14 }}=k \int_{M^{N=3}} \int_{M^{11}}\left(\delta^{11}(y)\right)=k \int_{M^{N=3}} \longrightarrow \\
k \frac{4 \pi R^{3}}{3},
\end{gathered}
$$




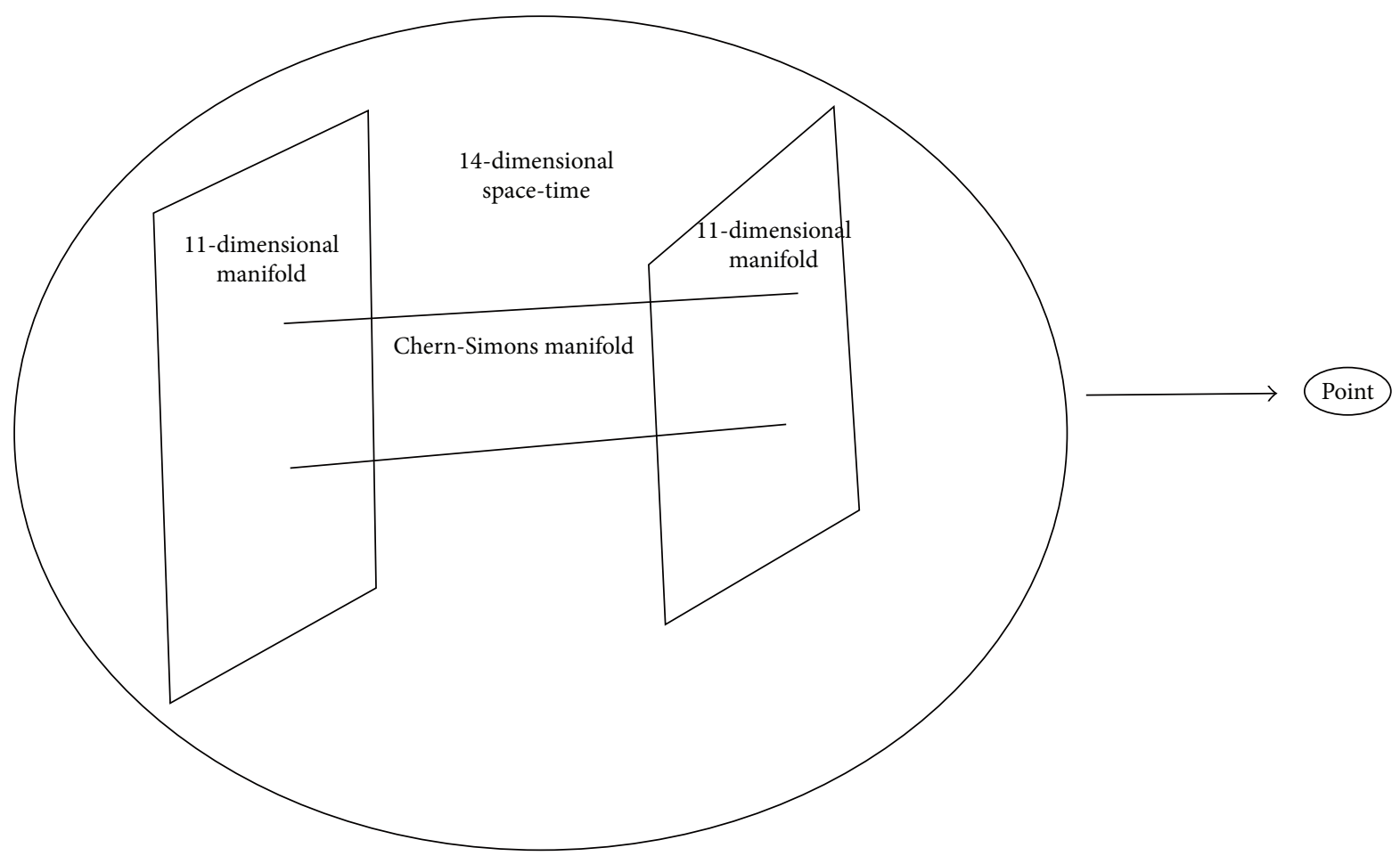

FIgURE 2: 14-dimensional manifold shrinks to one point.

where $R$ is the radius of manifold. By redefining scalar strings, we are able to show that the action of supergravity shrinks to one.

$$
\begin{aligned}
X & \longrightarrow\left(k \frac{4 \pi R^{3}}{3}\right)^{-1 / 11} X \\
\bar{X} & \longrightarrow\left(k \frac{4 \pi R^{3}}{3}\right)^{-1 / 11} \bar{X} \Longrightarrow \\
S_{\text {SUGRA-14 }} & =\left(k \frac{4 \pi R^{3}}{3}\right)^{-1} k \int_{M^{N=3}} \longrightarrow \\
\left(k \frac{4 \pi R^{3}}{3}\right)^{-1} k \frac{4 \pi R^{3}}{3} & =1 .
\end{aligned}
$$

This equation yields results that deserve our comments. In fact, two eleven-dimensional manifolds and the bulk interact with each other via different types of $C, G$, and Chern-Simons-fields. When we sum up supergravities that live on these manifolds and consider fields in the space between them, we get supergravity in 14-dimensional space. By canceling the anomaly in this new supergravity, we can obtain the relations between fields. By substituting these relations into the action of the 14-dimensional supergravity, we simply obtain one. This means that the 14-dimensional manifold with all its matter content can be topologically shrunk to one point (see Figure 2.). In fact, the system of the world began from this point and then expanded and constructed a 14-dimensional world similar to what happens in a Big Bang theory.

Another interesting result that comes out of our theory concerns the reduction of the action of the world to one. This means that the origin of all matter or fields is the same. In fact, at the first stage of the world, we have only a constant energy which lives on 14-dimensional manifold. Then, by internal interactions, two 11-dimensional manifolds are created. These manifolds interact with each other via an extra Chern-Simons manifold. By exchanging the energy between 11-dimensional manifolds, their energies are changed and two different actions are produced. Also, as due to interaction between strings, their shapes become different and different types of fields like spinors and bosons are emerged.

\section{Summary and Final Considerations}

In this paper, we have shown that the Chern-Simons terms of modified gravity may be understood as due to the interaction between two 11-dimensional manifolds in an $11+3$ dimensional space-time, where 3 is the dimension of a Lietype-algebra. We also argue that there is a direct relation between the dimension of the algebra and the dimension of the manifold. For example, for 11-dimensional world, the dimension of the Lie-algebra is three. If the rank of the fields which live in one manifold becomes larger than the rank of the algebra, there emerges an anomaly. This anomaly is produced as an effect of connecting fields in the bulk to fields which live in the manifold. To cancel this anomaly, we 
need to introduce another 11-dimensional manifold in the 11+3-dimensional space-time which interacts with the initial manifold by exchanging Chern-Simons terms. These ChernSimons terms produce an extra manifold. If we sum up the topology of the 11-dimensional manifolds and the topology of the Chern-Simons manifold, we can show that the total topology shrinks to one, which is consistent with predictions of the Big Bang theory.

Our proposal is actually another version of the $G$ theory in $[15,16]$. This approach removes anomalies in 11dimensional $M$-theory by a generalization of dimension to 14. In this research contribution, we have shown that the physics of an 11-dimensional spacing manifold plus a 3dimensional algebraic manifold is the same as the physics of 14-dimensional manifold. In fact, 11-dimensional $M$-theory is a free-anomaly theory, whenever a three-dimensional algebraic manifold is added to it. This 14-dimensional manifold can be broken to two 11-dimensional manifolds which are connected by a 3-dimensional Chern-Simons theory. Another interesting result that comes out is the reduction of the action of the world to one. This means that the origin of all matter or fields is the same. In fact, at the first stage of the world, we have only a constant energy which lives on 14-dimensional manifold. Then, by internal interactions, two 11-dimensional manifolds come out. These manifolds interact with each other via one extra Chern-Simons manifold. By exchanging the energy between 11-dimensional manifolds, their energies are changed and two different actions are produced. Also, as due to interaction between strings, their shapes differ and diverse types of fields like spinors and bosons emerge.

To conclude, we would like to point out that that all our treatment has been restricted to the purely bosonic sector of 11-dimensional supergravity, whose on-shell multiplet, besides the metric tensor and the 3-form gauge potential, includes the gravitino field. The latter has not been considered here; we have restricted ourselves to the bosonic sector. However, it would be a further task to inspect how the inclusion of the gravitino would affect our developments, possibly changing the dimension of the Lie algebra to be added to the 11-dimensional manifold. By including the fermion, it is no longer ensured that the local supersymmetry of the $11+3$-dimensional supergravity action remains valid. The change in the dimension of the Lie algebra could, in turn, give rise to new terms, so that the Chern-Simons modified gravity would be further extended as a result of including the gravitino. We intend to pursue an investigation on this issue and to report on it elsewhere.

In our model, the three-Lie algebra that we add, to pass from 11 to $14 D$, is geometrical. It is shown that all anomalies which are produced by axial bosonic fields in 10- and 11dimensional theories can be removed on 14-dimensional manifold. Maybe, a question arises: "which is the effect of fermionic anomalies on the number of dimensions?" It must be clear that this paper is the first step for solving anomalies in 11-dimensional theories. In the next step, by applying the model of this paper for fermions, the exact number of dimensions of the world can be estimated.

\section{Appendix}

\section{Details of Calculations}

In this section, we will bring details of calculations. First let us disclose more aspects of (6):

$$
\begin{aligned}
& I_{J}=\epsilon_{J} I=\epsilon_{J} \frac{\epsilon_{\alpha J}^{\alpha}+\Gamma_{\alpha J}^{\alpha}}{\epsilon_{\alpha J}^{\alpha}+\Gamma_{\alpha J}^{\alpha}} \\
& X^{I_{i}}=y^{I_{i}}+A^{I_{i}}+\epsilon^{I_{i}} \phi-\epsilon^{I_{i} J} I_{J} \\
& =y^{I_{i}}+A^{I_{i}}+\epsilon^{I_{i}} \phi-\epsilon^{I_{i} J} \epsilon_{J} \frac{\epsilon_{\alpha J}^{\alpha}+\Gamma_{\alpha J}^{\alpha}}{\epsilon_{\alpha J}^{\alpha}+\Gamma_{\alpha J}^{\alpha}} \\
& =y^{I_{i}}+A^{I_{i}}+\epsilon^{I_{i}} \phi-\epsilon^{I_{i} J} \Gamma_{\alpha J}^{\alpha}-\epsilon^{I_{i} J} \sum_{n=1}^{\infty}\left(\Gamma_{\alpha J}^{\alpha}\right)^{-n}+\cdots \\
& \left\{X^{I_{i}}, X^{I_{j}}\right\}=\sum_{I_{i}, I_{j}} \epsilon^{I_{i}^{\prime} I_{j}^{\prime}} \frac{\partial X^{I_{i}}}{\partial y^{I_{j}^{\prime}}} \frac{\partial X^{I_{j}}}{\partial y^{I_{j}^{\prime}}} \\
& =\sum_{I_{i}, I_{j}} \epsilon^{I_{i} I_{i}^{\prime}}\left(\partial_{I_{i}^{\prime}} A^{I_{j}}-\partial_{I_{i}^{\prime}}\left(\epsilon^{I_{j} I_{k}} \Gamma_{\alpha I_{k}}^{\alpha}\right)+\partial^{I_{i}} \phi \partial_{I_{j}} \phi+\cdots\right) \\
& =F^{I_{i} I_{j}}-R^{I_{i} I_{j}}+\partial^{I_{i}} \phi \partial_{I_{j}} \phi-\frac{1}{2} \epsilon^{I_{i} I_{j} I_{k} I_{m}} \phi R_{I_{i} I_{j} I_{k} I_{m}} \\
& \quad+\cdots,
\end{aligned}
$$

where $\phi$ is the Chern-Simons scalar field, $A^{I}$ is the gauge field, $\Gamma$ is related to the curvature $(R)$, and $I$ is a unit vector in the direction of the coordinate which can be expanded in terms of derivatives of metric.

Also, it is needed to reobtain the results of (10) with doing some mathematical calculations:

$$
\begin{aligned}
& \int_{M^{11}+M^{N=3}} \sqrt{g} C G G \\
& =\int_{M^{11}+M^{N=3}} \sqrt{g} \epsilon_{I_{1} I_{2} I_{3} I_{4} I_{1}^{\prime} I_{2}^{\prime} I_{3}^{\prime} I_{4}^{\prime} I_{5} I_{6} I_{7}} \epsilon^{\tilde{I}_{5} \tilde{I}_{6} \tilde{I}_{7}}\left(\frac{\partial X^{I_{5}}}{\partial y^{I_{5}}}\right. \\
& \left.\cdot \frac{\partial X^{I_{6}}}{\partial y^{I_{6}}} \frac{\partial X^{I_{7}}}{\partial y^{I_{7}}}\right) G^{I_{1} I_{2} I_{3} I_{4}} G^{I_{1}^{\prime} I_{2}^{\prime} I_{3}^{\prime} I_{4}^{\prime}} \\
& =\int_{M^{11}+M^{N=3}} \sqrt{g} \epsilon_{I_{1} I_{2} I_{3} I_{4} I_{1}^{\prime} I_{2}^{\prime} I_{3}^{\prime} I_{4}^{\prime} I_{5} I_{6} I_{7}} \epsilon^{\tilde{I}_{5} \tilde{I}_{6} \tilde{I}_{7}}\left(\frac{\partial X^{I_{5}}}{\partial y^{I_{5}}}\right. \\
& \left.\cdot \frac{\partial X^{I_{6}}}{\partial y^{\widetilde{I}_{6}}} \frac{\partial X^{I_{7}}}{\partial y^{\widetilde{I}_{7}}}\right) \times\left(\epsilon^{\widetilde{I}_{1} \tilde{I}_{2} \widetilde{I}_{3} \widetilde{I}_{4}} \frac{\partial X^{I_{1}}}{\partial y^{\widetilde{I}_{1}}} \frac{\partial X^{I_{2}}}{\partial y^{\widetilde{I}_{2}}} \frac{\partial X^{I_{3}}}{\partial y^{\widetilde{I}_{3}}} \frac{\partial X^{I_{4}}}{\partial y^{\widetilde{I}_{4}}}\right) \\
& \cdot\left(\epsilon^{\widetilde{I}_{1} \widetilde{I}_{2}^{\prime} \widetilde{I}_{3}^{\prime} \widetilde{I}_{4}^{\prime}} \frac{\partial X^{I_{1}^{\prime}}}{\partial \widetilde{I}_{1}^{\prime}} \frac{\partial X^{I_{2}^{\prime}}}{\partial y^{\widetilde{I}_{2}^{\prime}}} \frac{\partial X^{I_{3}^{\prime}}}{\partial y^{\widetilde{I}_{3}^{\prime}}} \frac{\partial X^{I_{4}^{\prime}}}{\partial y^{\widetilde{I}_{4}^{\prime}}}\right) \\
& =\int_{M^{11}+M^{N=3}} \sqrt{g} \epsilon_{I_{1} I_{2} I_{3} I_{4} I_{1}^{\prime} I_{2}^{\prime} I_{3}^{\prime} I_{4}^{\prime} I_{5} I_{6} I_{7}} \epsilon^{\tilde{I}_{5} \tilde{I}_{6} \tilde{I}_{7}}\left(\frac{\partial X^{I_{5}}}{\partial y^{I_{5}}}\right.
\end{aligned}
$$

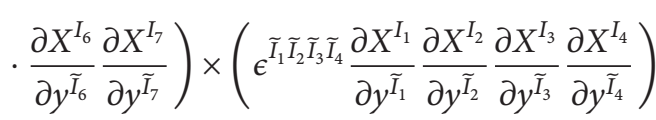


Advances in High Energy Physics

11

$$
\begin{aligned}
& \cdot\left(\epsilon^{\widetilde{I}_{1} \widetilde{I}_{2}^{\prime} \widetilde{I}_{3}^{\prime} \widetilde{I}_{4}^{\prime}} \frac{\partial X^{I_{1}^{\prime}}}{\partial y^{\widetilde{I}_{1}^{\prime}}} \frac{\partial X^{I_{2}^{\prime}}}{\partial \widetilde{I}_{2}^{\prime}} \frac{\partial X^{I_{3}^{\prime}}}{\partial y^{\widetilde{I}_{3}^{\prime}}} \frac{\partial X^{I_{4}^{\prime}}}{\partial y^{\widetilde{I}_{4}^{\prime}}}\right) \\
& =\int_{M^{11}+M^{N=3}} \sqrt{g} \epsilon_{I_{1} I_{2} I_{3} I_{4} I_{1}^{\prime} I_{2}^{\prime} I_{3}^{\prime} I_{4}^{\prime} I_{5} I_{6} I_{7}}\left(\epsilon^{\widetilde{I}_{4} \widetilde{I}_{5} \widetilde{I}_{7}} \frac{\partial X^{I_{4}}}{\partial y^{\widetilde{I}_{4}}}\right. \\
& \left.-\partial_{I_{i}} \phi \partial^{I_{j}} \phi\right)-\frac{1}{2} \int_{M^{11}+M^{N=3}} \sqrt{g}\left(\phi \epsilon_{I_{k} I_{m} I_{j}}^{I_{I}} F^{I_{k} I_{m}} F_{I_{i} I_{j}}\right) \\
& +\cdots \text {. }
\end{aligned}
$$

$$
\begin{aligned}
& \left.\cdot \frac{\partial X^{I_{5}}}{\partial y^{\widetilde{I}_{5}}} \frac{\partial X^{I_{7}}}{\partial \widetilde{I}_{7}}\right)\left(\epsilon^{\widetilde{I}_{4} \widetilde{I}_{6}} \frac{\partial X^{I_{4}^{\prime}}}{\partial \widetilde{I}_{4}^{\prime}} \frac{\partial X^{I_{6}}}{\partial \tilde{I}_{6}}\right) \times\left(\epsilon^{\widetilde{I}_{1} \widetilde{I}_{2}} \frac{\partial X^{I_{1}}}{\partial y^{\widetilde{I}_{1}}}\right. \\
& \left.\cdot \frac{\partial X^{I_{2}}}{\partial y^{\widetilde{I}_{2}}}\right)\left(\epsilon^{\widetilde{I}_{1} \tilde{I}_{2}^{\prime}} \frac{\partial X^{I_{1}^{\prime}}}{\partial y^{\widetilde{I}_{1}^{\prime}}} \frac{\partial X^{I_{2}^{\prime}}}{\partial \widetilde{I}^{\prime}}\right)\left(\epsilon^{\widetilde{I}_{3} \widetilde{I}_{3}} \frac{\partial X^{I_{3}}}{\partial \widetilde{I}^{I_{3}}} \frac{\partial X^{I_{3}^{\prime}}}{\partial y^{\widetilde{I}_{3}^{\prime}}}\right) \\
& =\int_{M^{11}+M^{N=3}} \sqrt{g}\left(\frac{1}{2} \epsilon^{I_{i} I_{j} I_{k} I_{m}} \phi R_{I_{k} I_{m} I_{l} I_{n}} R_{I_{i} I_{j}}^{I_{1} I_{n}}\right.
\end{aligned}
$$$$
\delta \int_{M^{11}+M^{N=3}} \sqrt{g} C G G
$$$$
=\delta \int_{M^{11}+M^{N=3}} \sqrt{g} \epsilon_{I_{1} I_{2} I_{3} I_{4} I_{1}^{\prime} I_{2}^{\prime} I_{3}^{\prime} I_{4}^{\prime} I_{5} I_{6} I_{7}} \epsilon^{\widetilde{I}_{5} \widetilde{I}_{6} \widetilde{I}_{7}}\left(\frac{\partial X^{I_{5}}}{\partial y^{\widetilde{I}_{5}}} \frac{\partial X^{I_{6}}}{\partial y^{\widetilde{I}_{6}}} \frac{\partial X^{I_{7}}}{\partial y^{\widetilde{I}_{7}}}\right) G^{I_{1} I_{2} I_{3} I_{4}} G^{I_{1}^{\prime} I_{2}^{\prime} I_{3}^{\prime} I_{4}^{\prime}}
$$$$
=\delta \int_{M^{11}+M^{N=3}} \sqrt{g} \epsilon_{I_{1} I_{2} I_{3} I_{4} I_{1}^{\prime} I_{2}^{\prime} I_{3}^{\prime} I_{4}^{\prime} I_{5} I_{6} I_{7}} \epsilon^{\widetilde{I}_{5} \widetilde{I}_{6} \widetilde{I}_{7}}\left(\frac{\partial X^{I_{5}}}{\partial y^{\widetilde{I}_{5}}} \frac{\partial X^{I_{6}}}{\partial y^{\widetilde{I}_{6}}} \frac{\partial X^{I_{7}}}{\partial \tilde{I}_{7}}\right)
$$$$
\times\left(\epsilon^{\widetilde{I}_{1} \tilde{I}_{2} \widetilde{I}_{3} \widetilde{I}_{4}} \frac{\partial X^{I_{1}}}{\partial y^{\widetilde{I}_{1}}} \frac{\partial X^{I_{2}}}{\partial y^{\widetilde{I}_{2}}} \frac{\partial X^{I_{3}}}{\partial y^{\widetilde{I}_{3}}} \frac{\partial X^{I_{4}}}{\partial y^{\widetilde{I}_{4}}}\right)\left(\epsilon^{\widetilde{I}_{1}^{\prime} \widetilde{I}_{2} \widetilde{I}_{3} \widetilde{T}_{4}} \frac{\partial X^{I_{1}^{\prime}}}{\partial y^{\widetilde{I}_{1}^{\prime}}} \frac{\partial X^{I_{2}^{\prime}}}{\partial y^{\widetilde{I}_{2}^{\prime}}} \frac{\partial X^{I_{3}^{\prime}}}{\partial y^{\widetilde{I}_{3}^{\prime}}} \frac{\partial X^{I_{4}^{\prime}}}{\partial y^{\widetilde{I}_{4}^{\prime}}}\right)
$$$$
=\int_{M^{10}+M^{N=3}} \sqrt{g} \epsilon_{I_{1} I_{2} I_{3} I_{4} I_{1}^{\prime} I_{2}^{\prime} I_{3}^{\prime} I_{4}^{\prime} I_{5} I_{6}} \epsilon^{\widetilde{I}_{5} \widetilde{I}_{6}}\left(\frac{\partial X^{I_{5}}}{\partial y^{\widetilde{I}_{5}}} \frac{\partial X^{I_{6}}}{\partial y^{\widetilde{I}_{6}}}\right)
$$$$
\times\left(\epsilon^{\widetilde{I}_{1} \widetilde{I}_{2} \widetilde{I}_{3} \widetilde{I}_{4}} \frac{\partial X^{I_{1}}}{\partial y^{\widetilde{I}_{1}}} \frac{\partial X^{I_{2}}}{\partial y^{\widetilde{I}_{2}}} \frac{\partial X^{I_{3}}}{\partial y^{\widetilde{I}_{3}}} \frac{\partial X^{I_{4}}}{\partial y^{\widetilde{I}_{4}}}\right)\left(\epsilon^{\widetilde{I}_{1} \tilde{T}_{2} \widetilde{I}_{3} \widetilde{T}_{4}} \frac{\partial X^{I_{1}^{\prime}}}{\partial y^{\widetilde{I}_{1}^{\prime}}} \frac{\partial X^{I_{2}^{\prime}}}{\partial y^{\prime}} \frac{\partial X^{I_{3}^{\prime}}}{\partial y^{\prime}} \frac{\partial X^{I_{4}^{\prime}}}{\partial y^{\widetilde{I}_{4}^{\prime}}}\right)
$$$$
=\int_{M^{10}+M^{N=3}} \sqrt{g} \epsilon_{I_{1} I_{2} I_{3} I_{4} I_{1}^{\prime} I_{2}^{\prime} I_{3}^{\prime} I_{4}^{\prime} I_{5} I_{6}}\left(\epsilon^{\tilde{I}_{4} \widetilde{I}_{5}} \frac{\partial X^{I_{4}}}{\partial y^{\widetilde{I}_{4}}} \frac{\partial X^{I_{5}}}{\partial \widetilde{I}_{5}}\right)\left(\epsilon^{\widetilde{I}_{4}^{\prime} \widetilde{I}_{6}} \frac{\partial X^{I_{4}^{\prime}}}{\partial y^{\widetilde{I}_{4}^{\prime}}} \frac{\partial X^{I_{6}}}{\partial y^{\widetilde{I}_{6}}}\right)
$$$$
\times\left(\epsilon^{\widetilde{I}_{1} \widetilde{I}_{2}} \frac{\partial X^{I_{1}}}{\partial y^{\widetilde{I}_{1}}} \frac{\partial X^{I_{2}}}{\partial y^{\widetilde{I}_{2}}}\right)\left(\epsilon^{\widetilde{I}_{1} \widetilde{I}_{2}^{\prime}} \frac{\partial X^{I_{1}^{\prime}}}{\partial \widetilde{I}_{1}^{\prime}} \frac{\partial X^{I_{2}^{\prime}}}{\partial y^{\widetilde{I}_{2}^{\prime}}}\right)\left(\epsilon^{\widetilde{I}_{3} \widetilde{I}_{3}} \frac{\partial X^{I_{3}}}{\partial \widetilde{I}^{I_{3}}} \frac{\partial X^{I_{3}^{\prime}}}{\partial \widetilde{I}_{2}^{\prime}}\right)
$$$$
=\int_{M^{10}+M^{N=3}} \sqrt{g} \sum_{n=1}^{5}\left(\operatorname{tr} F^{n}-\operatorname{tr} R^{n}+\operatorname{tr}\left(F^{n} R^{5-n}\right)\right)
$$

$$
\begin{aligned}
& +\int_{M^{10}+M^{N=3}} \sqrt{g}\left(\sum_{n=1}^{5}\left(\operatorname{tr}\left(F^{n}\left(\partial^{I_{i}} \phi \partial_{I_{j}} \phi\right)^{5-n}\right)\right)+\operatorname{tr}\left(F^{n}\left(\epsilon^{I_{i} I_{j} I_{k} I_{m}} R_{I_{i} I_{j} I_{k} I_{m}} \phi\right)^{5-n}\right)\right) \\
& -\int_{M^{10}+M^{N=3}} \sqrt{g}\left(\sum_{n=1}^{5}\left(\operatorname{tr}\left(R^{n}\left(\partial^{I_{i}} \phi \partial_{I_{j}} \phi\right)^{5-n}\right)\right)+\operatorname{tr}\left(R^{n}\left(\epsilon^{I_{i} I_{j} I_{k} I_{m}} R_{I_{i} I_{j} I_{k} I_{m}} \phi\right)^{5-n}\right)\right)+\cdots
\end{aligned}
$$


The first line of this equation removes the anomaly on the 11dimensional manifold of (4); however, the second and third lines show that extra anomalies can emerge due to the ChernSimons fields. (24):

At this stage, we can consider the process of obtaining

$$
\begin{aligned}
& S_{\text {SUGRA-14 }}=\delta\left(\int_{M^{14}} C G G+\int_{M^{14}} \bar{C} G \bar{G}+\int_{M^{14}} C \bar{G} G\right. \\
& \left.+\int_{M^{14}} \bar{C} \bar{G} \bar{G}\right)=\int_{M^{14}} \sum_{n=1}^{5}\left(\operatorname{tr} F^{n}-\operatorname{tr} R^{n}+\operatorname{tr}\left(F^{n} R^{5-n}\right)\right) \\
& +\int_{M^{14}} \sum_{n=1}^{5} \sum_{J=0}^{n} \sum_{m=0}^{n-J}\left(\operatorname{tr} \bar{F}^{J} F^{m}-\operatorname{tr} \bar{R}^{J} R^{m}+\operatorname{tr}\left(\bar{F}^{J} \bar{R}^{5-J} F^{m} R^{5-m}\right)\right) \\
& +\int_{M^{14}} \sum_{n=1}^{5} \sum_{J=0}^{n} \sum_{m=0}^{n-J}\left(\operatorname{tr} \bar{F}^{m} F^{J}-\operatorname{tr} \bar{R}^{m} R^{J}+\operatorname{tr}\left(\bar{F}^{m} \bar{R}^{5-m} F^{J} R^{5-J}\right)\right) \\
& +\int_{M^{14}} \sum_{n=1}^{5}\left(\operatorname{tr} \bar{F}^{n}-\operatorname{tr} \bar{R}^{n}+\operatorname{tr}\left(\bar{F}^{n} \bar{R}^{5-n}\right)\right) \\
& +\int_{M^{10}+M^{N=3}}\left(\sum_{n=1}^{5}\left(\operatorname{tr}\left(F^{n}\left(\partial^{I_{i}} \phi \partial_{I_{j}} \phi\right)^{5-n}\right)\right)\right. \\
& \left.+\operatorname{tr}\left(F^{n}\left(\epsilon^{I_{i} I_{j} I_{k} I_{m}} R_{I_{i} I_{j} I_{k} I_{m}} \phi\right)^{5-n}\right)\right) \\
& +\int_{M^{10}+M^{N=3}}\left(\sum_{n=1}^{5}\left(\operatorname{tr}\left(\bar{F}^{n}\left(\partial^{I_{i}} \bar{\phi} \partial_{I_{j}} \bar{\phi}\right)^{5-n}\right)\right)\right. \\
& \left.+\operatorname{tr}\left(\bar{F}^{n}\left(\epsilon^{I_{i} I_{j} I_{k} I_{m}} \bar{R}_{I_{i} I_{j} I_{k} I_{m}} \bar{\phi}\right)^{5-n}\right)\right)+\cdots \approx \int_{M^{14}} \sum_{n=1}^{5}(F+\bar{F})^{n} \\
& -\int_{M^{14}} \sum_{n=1}^{5}(R+\bar{R})^{n}+\int_{M^{14}} \sum_{n=1}^{5}(R \bar{F}+\bar{R} F)^{n}+\cdots \\
& \approx \int_{M^{14}} \sum_{n=1}^{5}\left(\left\{X^{I_{i}}, X^{I_{j}}\right\}+\left\{\bar{X}^{I_{i}}, \bar{X}^{I_{j}}\right\}\right)^{n}=0 \longrightarrow \\
& \left\{X^{I_{i}}, X^{I_{j}}\right\}=-\left\{\bar{X}^{I_{i}}, \bar{X}^{I_{j}}\right\} \longrightarrow \\
& X^{I_{i}}=i \bar{X}^{I_{i}} \longrightarrow \\
& y^{I_{i}}=i \bar{y}^{I_{i}} \\
& A^{I_{i}}=i \bar{A}^{I_{i}}, \\
& \phi=i \bar{\phi} .
\end{aligned}
$$

On the other hand, the result of (25) can be obtained as

$$
\begin{aligned}
& \int_{M^{11}+M^{N=3}} C G G \\
& =\int_{M^{11}+M^{N=3}} \epsilon^{I_{1} I_{2} I_{3} I_{4} I_{1}^{\prime} I_{2}^{\prime} I_{3}^{\prime} I_{4}^{\prime} I_{5} I_{6} I_{7}} \epsilon^{I_{5} I_{6} I_{7}}\left(\frac{\partial X^{I_{5}}}{\partial y^{I_{5}}} \frac{\partial X^{I_{6}}}{\partial y^{I_{6}}}\right. \\
& \left.\quad \cdot \frac{\partial X^{I_{7}}}{\partial y^{I_{7}}}\right) G_{I_{1} I_{2} I_{3} I_{4}} G_{I_{1}^{\prime} I_{2}^{\prime} I_{3}^{\prime} I_{4}^{\prime}} \epsilon^{I_{1} I_{2} I_{3} I_{4} I_{1}^{\prime} I_{2}^{\prime} I_{3}^{\prime} I_{4}^{\prime} I_{5} I_{6} I_{7}} \epsilon^{I_{5} I_{6} I_{7}}\left(\frac{\partial X^{I_{5}}}{\partial y^{I_{5}}} \frac{\partial X^{I_{6}}}{\partial y^{I_{6}}}\right.
\end{aligned}
$$

$$
\begin{aligned}
& \left.\cdot \frac{\partial X^{I_{7}}}{\partial y^{I_{7}}}\right) \times\left(\epsilon^{I_{1} I_{2} I_{3} I_{4}} \frac{\partial X^{I_{1}}}{\partial y^{I_{1}}} \frac{\partial X^{I_{2}}}{\partial y^{I_{2}}} \frac{\partial X^{I_{3}}}{\partial y^{I_{3}}} \frac{\partial X^{I_{4}}}{\partial y^{I_{4}}}\right) \\
& \cdot\left(\epsilon^{I_{1}^{\prime} I_{2}^{\prime} I_{3}^{\prime} I_{4}^{\prime}} \frac{\partial X^{I_{1}^{\prime}}}{\partial y_{1}^{I_{1}^{\prime}}} \frac{\partial X^{I_{2}^{\prime}}}{\partial y^{I_{2}^{\prime}}} \frac{\partial X^{I_{3}^{\prime}}}{\partial y^{I_{3}^{\prime}}} \frac{\partial X^{I_{4}^{\prime}}}{\partial y^{I_{4}^{\prime}}}\right) \\
& =\int_{M^{11}+M^{N=3}} \epsilon^{I_{1} I_{2} I_{3} I_{4} I_{1}^{\prime} I_{2}^{\prime} I_{3}^{\prime} I_{4}^{\prime} I_{5} I_{6} I_{7}} \epsilon^{I_{7} I_{4}}\left(\frac{\partial X^{I_{7}}}{\partial y^{I_{7}}}\right) \\
& \cdot\left(\epsilon^{I_{4} I_{5}} \frac{\partial X^{I_{4}}}{\partial y^{I_{4}}} \frac{\partial X^{I_{5}}}{\partial y^{I_{5}}}\right)\left(\epsilon^{I_{4}^{\prime} I_{6}} \frac{\partial X^{I_{4}^{\prime}}}{\partial y^{I_{4}}} \frac{\partial X^{I_{6}}}{\partial y^{I_{6}}}\right) \\
& \times\left(\epsilon^{I_{1} I_{2}} \frac{\partial X^{I_{1}}}{\partial y^{I_{1}}} \frac{\partial X^{I_{2}}}{\partial y^{I_{2}}}\right)\left(\epsilon^{I_{1}^{\prime} I_{2}^{\prime}} \frac{\partial X^{I_{1}^{\prime}}}{\partial y^{I_{1}^{\prime}}} \frac{\partial X^{I_{2}^{\prime}}}{\partial y^{I_{2}^{\prime}}}\right) \\
& \cdot\left(\epsilon^{I_{3} I_{3}^{\prime}} \frac{\partial X^{I_{3}}}{\partial y^{I_{3}}} \frac{\partial X^{I_{3}^{\prime}}}{\partial y^{I_{3}^{\prime}}}\right)=k \int_{M^{N=3}} \int_{M^{11}}\left(\delta^{11}(y)\right. \\
& \left.+\sum_{n=1}^{6}\left(\frac{\partial X^{I}}{\partial y^{I}}\right)^{6-n}\left(F^{n}-R^{n}+\cdots\right)\right) \\
& =k \int_{M^{N=3}} \int_{M^{11}}\left(\delta^{11}(y)\right. \\
& \left.+\sum_{n=1}^{6}\left(\frac{\partial X^{I}}{\partial y^{I}}\right)^{6-n}\left(\left\{X^{I_{i}}, X^{I_{j}}\right\}\right)^{n}\right) \text {. }
\end{aligned}
$$

\section{Conflicts of Interest}

The authors declare that they have no conflicts of interest.

\section{Acknowledgments}

The work of Alireza Sepehri has been supported financially by the Research Institute for Astronomy and Astrophysics of Maragha (RIAAM), Iran, under Research Project no. 1/523775.

\section{References}

[1] R. Jackiw and S.-Y. Pi, "Chern-Simons modification of general relativity," Physical Review D: Particles, Fields, Gravitation, and Cosmology, vol. 68, Article ID 104012, 2003.

[2] B. Pereira-Dias, C. A. Hernaski, and J. A. Helayël-Neto, "Probing the effects of lorentz-symmetry violating chern-simons and ricci-cotton terms in higher derivative gravity," Physical Review D: Particles, Fields, Gravitation and Cosmology, vol. 83, no. 8, 2011.

[3] K. Konno, T. Matsuyama, and S. Tanda, "Does a black hole rotate in Chern-Simons modified gravity?" Physical Review D: Particles, Fields, Gravitation and Cosmology, vol. 76, no. 2, Article ID 024009, 2007.

[4] D. Guarrera and A. J. Hariton, "Papapetrou energy-momentum tensor for Chern-Simons modified gravity," Physical Review D: Particles, Fields, Gravitation and Cosmology, vol. 76, no. 4, 2007. 
[5] K. K. Nandi, I. R. Kizirgulov, O. V. Mikolaychuk, N. P. Mikolaychuk, and A. A. Potapov, "Quantum phase shift in ChernSimons modified gravity," Physical Review D: Particles, Fields, Gravitation and Cosmology, vol. 79, no. 8, Article ID 083006, 2009.

[6] P. J. Porfírio, J. B. Fonseca-Neto, J. R. Nascimento, A. Petrov, J. Ricardo, and A. Santos, "Chern-Simons modified gravity and closed timelike curves," Physical Review D: Particles, Fields, Gravitation and Cosmology, vol. 94, no. 4, 2016.

[7] S. Alexander and N. Yunes, "Chern-Simons modified general relativity," Physics Reports, vol. 480, no. 1-2, pp. 1-55, 2009.

[8] S. Chen and J. Jing, "Geodetic precession and strong gravitational lensing in dynamical Chern-Simons-modified gravity," Classical and Quantum Gravity, vol. 27, no. 22, 225006, 16 pages, 2010.

[9] N. Yunes and C. F. Sopuerta, "Perturbations of Schwarzschild black holes in Chern-Simons modified gravity," Physical Review D: Particles, Fields, Gravitation and Cosmology, vol. 77, no. 6, 2008.

[10] F. Canfora, A. Giacomini, and A. R. Zerwekh, "Kaluza-Klein theory in the limit of large number of extra dimensions," Physical Review D: Particles, Fields, Gravitation and Cosmology, vol. 80, no. 8, Article ID 084039, 2009.

[11] S. Wu, "General rotating charged Kaluza-Klein AdS black holes in higher dimensions," Physical Review D: Particles, Fields, Gravitation and Cosmology, vol. 83, no. 12, 2011.

[12] T. Houri and K. Yamamoto, "Killing-Yano symmetry of KaluzaKLEin black holes in five dimensions," Classical and Quantum Gravity, vol. 30, no. 7, 075013, 21 pages, 2013.

[13] T. Tatsuoka, H. Ishihara, M. Kimura, and K. Matsuno, "Extremal charged black holes with a twisted extra dimension," Physical Review D: Particles, Fields, Gravitation and Cosmology, vol. 85, no. 4, 2012.

[14] P. Horava and E. Witten, "Heterotic and Type I string dynamics from eleven dimensions," Nuclear Physics B, vol. 460, no. 3, Article ID 9510209, pp. 506-524, 1996.

[15] A. Sepehri and R. Pincak, "The birth of the universe in a new G-theory approach," Modern Physics Letters A, vol. 32, no. 5, Article ID 1750033, 1750033, 32 pages, 2017.

[16] A. Sepehri, A. Pradhan, R. Pincak, F. Rahaman, A. Beesham, and T. Ghaffary, "Birth of the GUP and its effect on the entropy of the universe in Lie- $N$-algebra," International Journal of Geometric Methods in Modern Physics, vol. 14, no. 9, Article ID 1750130, 2017.

[17] P. Horava and E. Witten, "Eleven-dimensional supergravity on a manifold with boundary," Nuclear Physics B, vol. 475, pp. 94$114,1996$.

[18] J. Bagger and N. Lambert, "Gauge symmetry and supersymmetry of multiple M2-branes," Physical Review D: Particles, Fields, Gravitation and Cosmology, vol. 77, no. 6, 065008, 6 pages, 2008.

[19] P. Ho and Y. Matsuo, "M5 from M2," Journal of High Energy Physics, vol. 2008, no. 06, p. 105, 2008. 

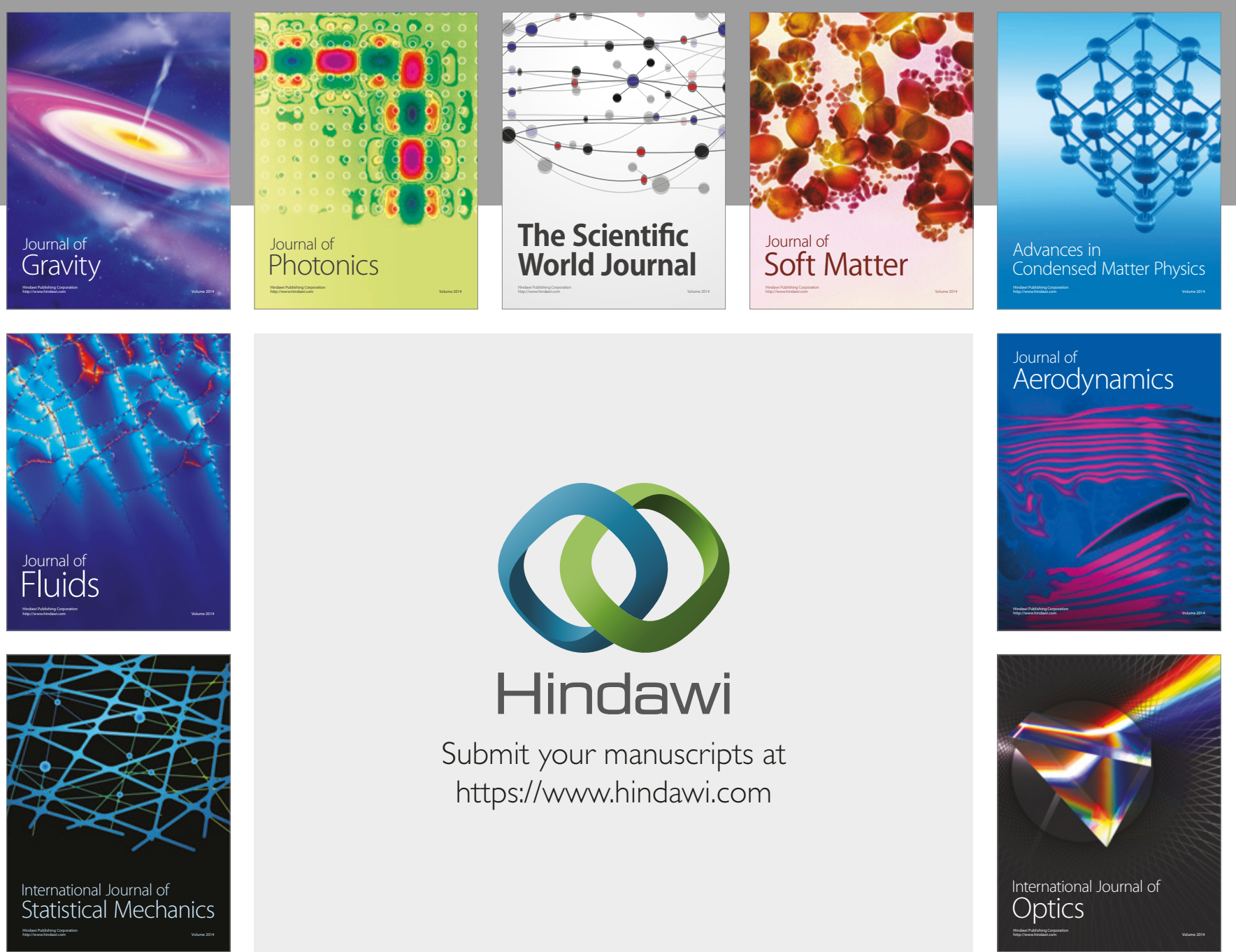

Submit your manuscripts at

https://www.hindawi.com
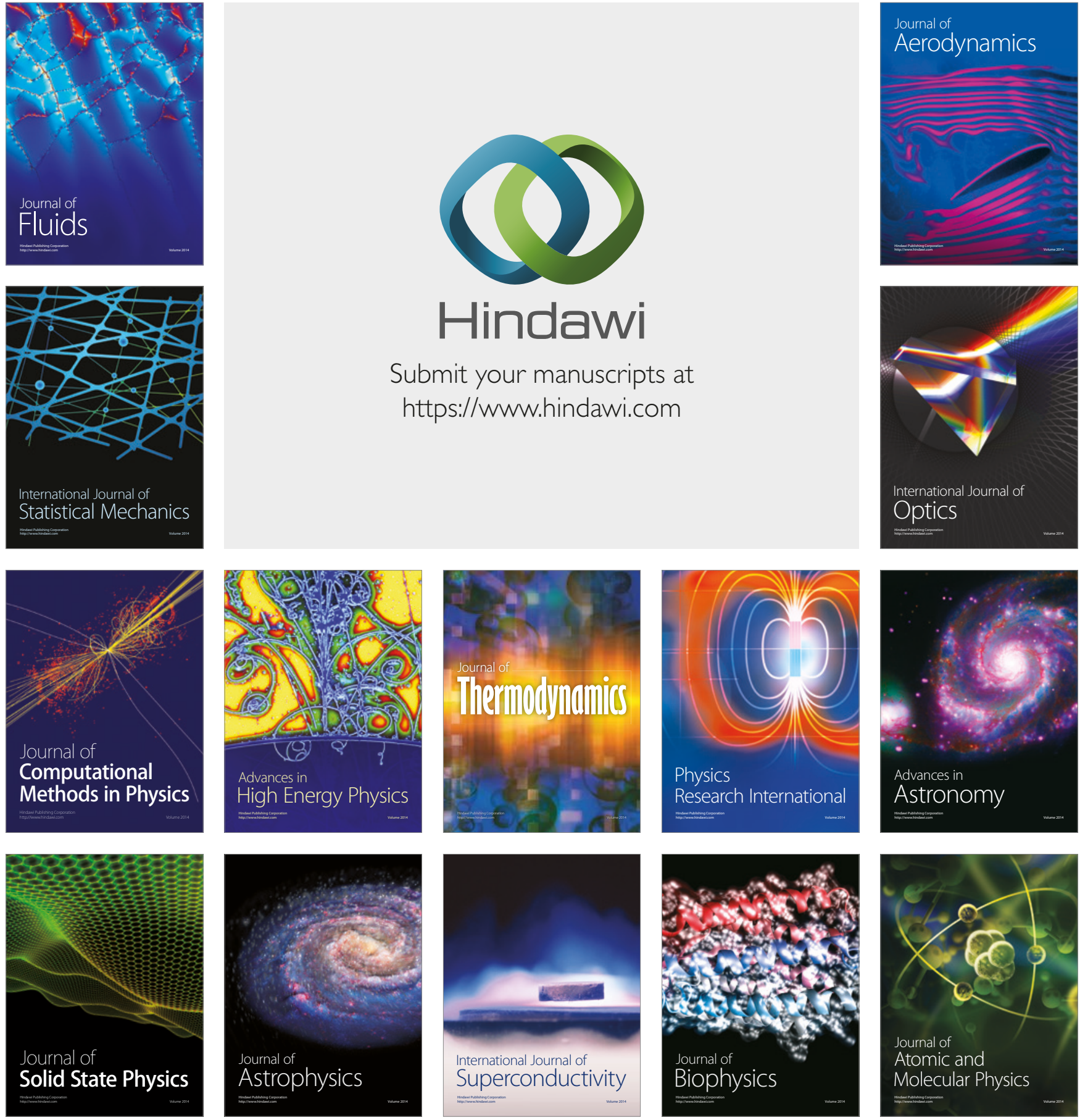